\title{
LAMOST Spectrograph Response Curves: Stability and Application to flux calibration
}

Bing Du ${ }^{1,2}$, A-Li Luo ${ }^{1,2}$, Zhong-Rui Bai ${ }^{1,2}$, Xiao Kong ${ }^{1}$, Jian-Nan Zhang ${ }^{1}$, Yan-Xin Guo 1, Neil James Cook ${ }^{3}$, Wen Hou ${ }^{1}$, Hai-Feng Yang ${ }^{1}$, Yin-Bi Li ${ }^{1}$, Yi-Han Song ${ }^{1}$, Jian-Jun Chen ${ }^{1}$, Fang Zuo ${ }^{1}$, Ke-Fei Wu ${ }^{1}$, Meng-Xin Wang ${ }^{1}$, You-Fen Wang ${ }^{1}$, and Yong-Heng Zhao ${ }^{1}$

Received _ _ accepted _

\footnotetext{
${ }^{1}$ Key Laboratory of Optical Astronomy, National Astronomical Observatories, Chinese Academy of Sciences, Beijing 100012, China. Ial@nao.cas.cn

${ }^{2}$ University of Chinese Academy of Sciences, Beijing 100049, China.

${ }^{3}$ Centre for Astrophysics Research, School of Physcis, Astronomy and Mathematics, University of Hertfordshire, College Lane, Hartfield AL10 9AB, UK.
} 


\begin{abstract}
The task of flux calibration for LAMOST (Large sky Area Multi-Object Spectroscopic Telescope) spectra is difficult due to many factors. For example, the lack of standard stars, flat fielding for large field of view, and variation of reddening between different stars especially at low galactic latitudes etc. Poor selection, bad spectral quality, or extinction uncertainty of standared stars not only might induce errors to the calculated spectral response curve (SRC), but also might lead to failures in producing final $1 \mathrm{D}$ spectra. In this paper, we inspected spectra with Galactic latitude $|b| \geq 60^{\circ}$ and reliable stellar parameters, determined through the LAMOST Stellar Parameter Pipeline (LASP), to study the stability of the spectrograph. To guarantee the selected stars had been observed by each fiber, we selected 37,931 high quality exposures of 29,000 stars from LAMOST DR2, and more than 7 exposures for each fiber. We calculated the SRCs for each fiber for each exposure, and calculated the statistics of SRCs for spectrographs with both the fiber variations and time variations. The result shows that the average response curve of each spectrograph (henceforth ASPSRC) is relatively stable with statistical errors $\leq 10 \%$. From the comparison between each ASPSRC and the SRCs for the same spectrograph obtained by 2D pipeline, we find that the ASPSRCs are good enough to use for the calibration. The ASPSRCs have been applied to spectra which were abandoned by LAMOST 2D pipeline due to the lack of standard stars, increasing the number of LAMOST spectra by 52,181 in DR2. Comparing those same targets with SDSS, the relative flux differences between SDSS spectra and that of LAMOST spectra with the ASPSRC method are less than $10 \%$, which underlines that the ASPSRC method is feasible for LAMOST flux calibration.
\end{abstract}


Subject headings: techniques: spectroscopic — methods: data analysis - methods: statistical 


\section{Introduction}

The LAMOST is a quasi-meridian reflecting Schmidt telescope with an effective aperture of $\sim 4 \mathrm{~m}$ and field of view (FoV) of 5 degree in diameter. At the focal plane, 4,000 robotic optical fibers of aperture size 3.3 arcsec projected on the sky relay the target light to 16 spectrographs, 250 fibers each (Cui et al. 2012; Deng et al. 2012). Proceeded by one-year Pilot Survey, the LAMOST Regular Surveys started in September 2012.

The wavelength range of LAMOST covers 3,700 to 9,000 $\AA$ and is recorded in two

arms, a blue arm (3,700-5,900 $\AA$ ) and a red arm (5,700-9,000 $\AA$ ), with the resolving power of $\mathrm{R} \sim 1,800$. A final spectrum is obtained by merging several exposures and connecting wavelength bands.

Raw data from the LAMOST surveys are reduced with the LAMOST 2D pipeline LuO et al. 2015). The procedures used by the 2D pipeline, similar to those of SDSS (Stoughton et al. 2002), aim to extract spectra from the CCD images and then calibrate them. The main tasks of the 2D pipeline include the steps of fiber tracing, flux extraction, wavelength calibration, flat fielding, sky subtraction, flux calibration, multi-exposure co-addition and the connection of the two wavelength bands. Since the data reduction steps are the reverse process of the data acqusition process, we should understand the data acquisition process of LAMOST, which can be simplified as follows.

$$
F_{o}(j, \lambda)=\left[F_{i}(j, \lambda) \times d_{s}(\lambda)+\operatorname{sky} y_{r}(\lambda)\right] \times d_{f}(\lambda) \times d_{p}(\lambda)+\operatorname{scatter}(j, \lambda)+C_{k}(j, \lambda)+B
$$

In this equation, $F_{o}(j, \lambda)$ is the observed signal, where $j$ denotes the $j$-th fiber and $\lambda$ denotes the wavelength;

$F_{i}(j, \lambda)$ is the target signal before pass through the atmosphere;

$d_{s}(\lambda)$ is the extinction function including atmospheric and interstellar reddening; 
$s k y_{r}(\lambda)$ is sky background;

$d_{f}(\lambda)$ is the fiber transmission function, a random number selected from a Gaussian distribution, with a mean of 0.9 and variance of 1.0 ;

$d_{p}(\lambda)$ is the spectral response function due to the dispersion of the spectrograph;

$\operatorname{scatter}(j, \lambda)$ is the scattering light including symmetrical scattering and the cross-contamination of fibers;

$C_{k}(j, \lambda)$ is the parameter to compensate with cosmic rays;

$B$ is the CCD background.

The purpose of the LAMOST flux calibration is to remove the spectral response curve (SRC) from observations. Considering that $d_{f}(\lambda)$ is divided during the flat field, the SRCs of spectrographs could be simplified as shown in equation 2 , which only includes $d_{s}(\lambda)$ and $d_{p}(\lambda)$.

$$
S R C(j, \lambda)=d_{s}(\lambda) \times d_{p}(\lambda)
$$

In the real flux calibration process, $d_{s}(\lambda)$ and $d_{p}(\lambda)$ are considered as a whole SRC, by which the single exposure is divided. For the LAMOST 2D pipeline, selection of standard stars is the first step of flux calibration (Song et al. 2012). The pipeline selects standard stars automatically by comparing all the observed spectra with the KURUCZ library produced based on the ATLAS9 stellar atmosphere model data (Castelli et al. 2004). For each of the 16 spectrographs, several high quality spectra with $\mathrm{SNR} \geq 10,5,750$ $\mathrm{K} \leq T_{\text {eff }} \leq 7,250 \mathrm{~K}, \log \mathrm{g} \geq 3.5$ dex and $-1.0 \operatorname{dex} \leq[\mathrm{Fe} / \mathrm{H}] \leq 0$ dex are selected as standard stars. Actually, the LAMOST 2D pipeline picks out standards with the temperature in the range of $6,000-7,000 \mathrm{~K}$ at first step, if there is not enough stars in this range, the 2D pipeline will extend the range to $5,750-7,250 \mathrm{~K}$. If more than 3 standard stars are found for a spectrograph, the SRCs of the spectrograph can be derived by comparing the 
observed spectra with synthetic spectra (using the corresponding parameters from the KURUCZ spectral library). Because the 2D pipeline estimates the parameters by simple fitting with KURUCZ model, the parameters have great uncertainties for the stars with $[\mathrm{Fe} / \mathrm{H}]<-1.0$ dex, meanwhile considering that the number of metal poor stars is small in each spectrograph, the $2 \mathrm{D}$ pipeline uses the metallicity cut of $-1.0 \mathrm{dex} \leq[\mathrm{Fe} / \mathrm{H}] \leq 0$ dex for the selection of the standards. Unfortunately, for the current LAMOST 2D pipeline, when there are not enough suitable standard stars, data reduced of the spectrograph has to be suspended.

In this paper, to rescue the unsuccessful spectra, we propose a novel flux calibration method based on the stability analysis of the SRCs. Thanks to more than 2 million spectra, with reliable stellar parameters in DR2, we are able to statistically measure the instrument stability. Through stellar parameters, the SRC of each fiber could be obtained. By averaging SRCs in each spectrograph, we can get an average spectrograph SRC (ASPSRC), and use it to calibrate spectra in each spectrograph without pre-selecting the flux standard stars assuming the ASPSRC is sufficiently stable. This flux calibration method can rescue more spectra from LAMOST which were abandoned by 2D pipeline.

The paper is organized as follows. Section 2 details of the procedures used to create the ASPSRC for each spectrograph. The accuracy analysis of the ASPSRC and its application to flux calibration are presented in Section 3. We conclude with Section 4 which summarizes and discusses the results. 


\section{Statistical Spectrograph Response Curves}

\subsection{Selection of the Sample}

Work by Xiang et al.(2015) show that variations of the SRCs exist, this is done by using stars in high dense fields, however these suffer from high interstellar extinction. However, to study the variations of the SRCs one should use stars with less extinction. Therefore, we selected stars at high Galactic latitude to analyze instrument response (Fitzpatrick et al. 1999, 2007).

To obtain a good approximation of the ASPSRCs, we require as many flux standard stars possible. To ensure the quality of the sample, the stellar parameters of LASP (Wu et al. 2011a b) were used to select the F-stars with the highest signal to noise ratios (SNRs). We selected stars with $6,000 \mathrm{~K} \leq T_{\text {eff }} \leq 7,000 \mathrm{~K}, \log \mathrm{g} \geq 3.5$ dex and Galactic latitude $|b| \geq 60^{\circ}$. This resulted in 29,526 targets with 37,931 pairs (blue and red arm ) of single exposures. For the ASPSRC, we have enough stars in the temperature range $6,000 \mathrm{~K} \leq T_{\text {eff }} \leq 7,000 \mathrm{~K}$, so we don't need to extend to $5,750 \mathrm{~K} \leq T_{\text {eff }} \leq 7,250 \mathrm{~K}$ as the 2D pipeline does. Metallicity mainly affect the blue-arm spectra at wavelengths less than $4,500 \AA$. An error of $0.2 \mathrm{dex}$ in $[\mathrm{Fe} / \mathrm{H}]$ can change the SED shape between $3,800 \AA$ and 4,500 $\AA$ by approximately $3 \%$, while the effects at wavelengths greater than 4,500 $\AA$ are only marginally (Xiang et al. 2015). As the ASPSRCs are derived from a great number of standard stars instead of a group of several standards in the 2D pipeline, and $90 \%$ of the metallicities are in the range of $[\mathrm{Fe} / \mathrm{H}] \geq-1.0$ dex, which the averaged SRCs are generated from. The accuracy of the parameters measured by LASP are good enough even for metal poor stars, and will not affect the averaged result. Thus we did not use a metallicity cut in this sample selection.

With the benefit of the large sample of targets that satisfied the above parameter 
space, we find that there are sufficient and appropriate exposures across all fibers and spectrographs to allow us to use them as standards. Fig 1 shows the histogram of the number of standards per spectrograph from DR2, which indicates that at least 7 standards per fiber (with 250 fibers in each spectrograph this is equivalent to at least 1,750 individual exposures, shown in Fig 11. Fig 2 shows the histogram of their effective temperatures, mostly located in the vicinity of $6,100 \mathrm{~K}$ (i.e. F8 type stars).

\subsection{Spectral Response Curves}

Let $F_{o}(\lambda)$ and $F_{i}(\lambda)$ denote the measured and intrinsic spectral flux density thus,

$$
F_{o}(\lambda)=d_{s}(\lambda) d_{p}(\lambda) F_{i}(\lambda)
$$

where $d_{s}(\lambda)$ is the combined atmospheric and interstellar extinction, and $d_{p}(\lambda)$ the telescope and instrumental response. In this work, we adopted synthetic flux as $F_{i}(\lambda)$, which is calculated using SPECTRUM based on the ATLAS9 stellar atmosphere model data released by Fiorella Castelli. The synthetic spectra of $1 \AA$ dispersion from the library of KURUCZ were used, then the spectra were degraded to the LAMOST spectral resolution by convolving with a Gaussian function. Only those with a constant micro-turbulent velocity of $2.0 \mathrm{~km} / \mathrm{s}$ and a zero rotation velocity were adopted, since these two parameters

have little effect on the spectral energy distribution (SED) at a given temperature (Grupp 2004).

The interstellar extinction can be neglected owing to our selection of high latitude standards, however the atmospheric extinction can not be separated from instrumental response. The SRCs in this paper include atmospheric extinction, and their variations are included in the uncertainty of the SRCs.

It is generally assumed that the SRCs are smooth functions of wavelength. In order 
to derive the SRCs, we applied a low-order piecewise polynomial fitting to the ratios of the observed and the synthetic spectra of the standard stars. Fig 3 shows examples of the SRC fitting for one fiber in both arms. For each standard star, the blue- and red-arm spectra were divided into five and six wavelength regions respectively, and each region was fitted with a second- or third-order polynomial, which are represented by the thick colored lines in Fig 3. The piecewise polynomials were derived through minimizing $\mid$ synthetic $\times$ polynomial - observed $\mid$. We defined a series of clean spectral regions avoiding the prominent stellar absorption features and the telluric absorption bands. The fitted polynomial values of data in these clean regions is indicated by asterisks in Fig 3, which were used for the final SRC fitting. The wavelengths of the join points were fixed to space between $200 \AA$ for adjacent spectral regions, the overlaps were median filtered to join together the adjacent regions. The final SRCs are represented by the black curves in the inserts in Fig 3 .

\subsection{Derivation of the ASPSRCs}

For the 250 fibers in each spectrograph, at least 1800 good SRCs (through multiexposures) were derived (excluding the bad fibers). We chose the fitted SRCs rather than the direct ratios of observed and synthetic to estimate the ASPSRC because the direct ratios are susceptible to noise. We concentrate on the relative flux calibration rather than absolute flux calibration, such that, for a given spectrograph, SRCs yielded by the spectra of the individual standard stars were divided by the average of their SRCs (i.e. the SRCs were scaled to a mean value of unity). It is generally assumed that the differences in the sensitivity of the individual fibers are well corrected via flat-fielding and thus the 250 fibers of a given spectrograph share a single SRC. Accordingly, the SRCs of the fibers can be regarded as independent measurements of the SRC, thus the ASPSRC and uncertainties 
can be derived by traditional statistical methods. The means and standard deviations of the Gaussian functions in Fig 4 give three examples of the spectral response and uncertainty estimation at wavelength point 4,000 $, 4,500 \AA$, and 5,000 $\AA$ of spectrograph No.1. All wavelength points contribute to the final ASPSRC for a spectrograph, and the red curves in Fig 5, Fig 6, Fig 7 and Fig 8 show the blue and red-arm ASPSRCs of the 16 spectrographs.

We expect that there is a unified response for a given spectrograph during exposures of different times and using different plates. The red curves in Fig 5, Fig 6, Fig 7 and Fig 8 are more likely to be, at least, very close to the SRCs of the instrument including the atmospheric extinction, which means that the ASPSRCs derived can be used to calibrate the plates which lack standard stars. Some ASPSRCs drop deeply on the edges due to the very low sensitivities of the instrument, which is the most challenging problem for LAMOST flux calibration.

We calculated the mean values of absolute and relative uncertainties for $\mathrm{g}, \mathrm{r}$ and i-bands, which are presented in Table 1. Table 1 shows that for all 16 spectrographs, the uncertainties are smaller than $8 \%$ for both $\mathrm{g}$ and $\mathrm{i}$-bands. The $\mathrm{r}$-band is located at the edge of both arms and thus due to the low sensitivities, the uncertainties for $\mathrm{r}$-band are much larger ( for example Spectrograph No.5 can differ by up to $11.13 \%$. This means the fluxes and centroids of the lines located at the junction of the blue and red arms (such as $\mathrm{Na} \mathrm{D}$ at $\lambda 5,892 \AA)$, are sometimes not credible.

\subsection{Time Variations}

Generally, the LAMOST observational season spans nine months from September to next June. The DR2 collected the observed data from October 2011 to June 2014, and there are nine quarters totally (about 3 consecutive months for a quarter). We calculated 
ASPSRCs for each quarter (hereafter called Quarter ASPSRC to distinguish from DR2 ASPSRC), and compared these nine Quarter ASPSRCs with the DR2 ASPSRC for each spectrograph. The distributions of residual between the nine Quarter ASPSRCs and the DR2 ASPSRC are shown in Fig 9, Fig 10, Fig 11 and Fig 12 (blue arm in left panel and red arm in right panel). The figures show that there are not obvious gradual and systematic errors along with time. Still, we can conclude that spectrograph No.4, No.11, No.15 and No.16 are more stable than others during the DR2 period.

\section{Flux Calibration Based on ASPSRCs}

The spectral flux calibration of target objects is generally achieved through obtaining separate measurements of spectrophotometric standard stars (Oke et al. 1990; Hamuy et al. 1992, 1994) on the same observing night with the same instrumental setup. However large spectroscopic survey, obtaining separate measurements of sufficient standard stars for each night and each spectrograph becomes impossible, and an alternative strategy has to be adopted. In the case of the Sloan Digital Sky Survey (York et al. 2000), F turn-off stars within the FoV are used to calibrate the spectra. These standards are preselected based

on the photometric colors and are observed simultaneously with the targets Stoughton et al. 2002; Yanny et al. 2009). The intrinsic SEDs of F turn-off stars are well determined by theoretical models of stellar atmospheres and the effects of interstellar extinction can be characterized and removed using the all-sky extinction map of Schlegel et al. (1998) (Schlegel et al. 1998; Schlafly et al. 2010). Without a photometric system for LAMOST, and lacking of extinction values especially for low galactic latitudes, the standard stars are not pre-assigned. Usually, the flux standard stars are selected from the spectra in each spectrograph after observation. Sometimes, the selection of the standard stars fails, thus the spectrograph of plate has to be abandoned by the LAMOST 2D pipeline. This is indeed 
why the ASPSRC method is important, as using fixed instrumental response curves can recover some of these abandoned plates.

\subsection{Co-add the multi-exposures}

To improve the SNRs and overcome the effect of cosmic rays, each field is designed to be exposed multiple times. The spectra of each single exposure may be on different scales due to the variation of observational condition. The spectra on different scales can not be co-added since they are divided by the same ASPSRC. Fig 13 shows the spectra of 6 exposures. Not only are the scales of exposures different but also the scales of the two arms are discrepant. For the LAMOST 2D pipeline, the single exposure spectra are scaled to the median of the multi-exposures. Here we try to scale the blue and red band according to the photometry of the $\mathrm{g}$ and $\mathrm{i}$ band respectively.

The monochromatic AB magnitude is defined as the logarithm of a spectral flux density with a zero-point of 3631 Jansky (Oke et al. 1983), where 1 Jansky $=1$ Jy $=$ ${ }^{10^{-26}} \mathrm{WHz}^{-1} \mathrm{~m}^{-2}=10^{-23} \mathrm{ergs}^{-1} \mathrm{~Hz}^{-1} \mathrm{~cm}^{-2}$. If the spectral flux density is denoted $f_{\nu}$, the monochromatic $\mathrm{AB}$ magnitude is:

$$
m_{A B}(\nu)=-2.5 \log _{10} f_{\nu}-48.60
$$

Actual measurements are always made across some continuous range of wavelengths. The bandpass AB magnitude is defined similarly, and the zero point corresponds to a bandpass-averaged spectral flux density of 3631 Jansky.

$$
m_{A B}=-2.5 \log _{10}\left(\frac{\int f_{\nu}(h \nu)^{-1} e(\nu) d \nu}{\int 3631 J y(h \nu)^{-1} e(\nu) d \nu}\right)
$$

where $e(\nu)$ is the equal-energy filter response function. The $(h \nu)^{-1}$ term assumes that 
the detector is a photon-counting device such as a CCD or photomultiplier. The synthetic magnitude can be obtained by convolving the flux spectra with the SDSS g and i band transmission curves (Hamuy et al. 1992, 1994). We adopted the $\mathrm{g}$ and i filter zero points from Pickles (Pickles 2010). The spectra are then scaled by comparing the synthetic magnitude with the photometry magnitude. The scale coefficient $S C(g)$ and $S C(i)$ are obtained as follows, and are multiplied with observed spectra.

$$
S C(g)=10^{-0.4 \times\left[\operatorname{mag}(g)-\operatorname{mag}_{\text {synthetic }}(g)\right]} ; \quad S C(i)=10^{-0.4 \times\left[\operatorname{mag}(i)-\operatorname{mag}_{\text {synthetic }}(i)\right]}
$$

The spectra of Fig 13 were scaled using the method described above. The rescaled spectra can then be co-added, and the final spectra derived, which is shown in Fig 13 (bottom panel). It should be noted that this method is subject to the SNR of the spectra, since the synthetic magnitudes depend on the quality of the spectra.

This method needs the photometry magnitudes of $g$ and $\mathrm{i}$-band for each target, thus we cross-matched LAMOST targets with Pan-STARRS1 (Tonry et al. 2012) within 3 mas. The LAMOST sources are selected from multiple catalogs with multi-band photometry. Consequently, not all the LAMOST targets overlap Pan-STARRS1. By cross-matching, we found that about $80 \%$ of the LAMOST targets are included in Pan-STARRS1. For those targets not in the Pan-STARRS1 catalog, SDSS PetroMag ugriz had been adopted. About $60 \%$ of the LAMOST targets have the overlapping observations with SDSS. However, there are still about $10 \%$ of the LAMOST targerts neither included in Pan-STARRS1 nor in SDSS, and it is difficult to obtain their photometry in the optical band. For the LAMOST, the efficiencies of the blue and red-arms can not be corrected by flat fielding since the throughput of the two arms for each spectrograph are different and varies when the telescope pointing changes. The flat field of the two arms for each spectrograph are processed independently. Using photometry, we can avoid big scale jump between the two arms although there are some photometry errors. Without the reference of photometry, we 
have to only use the overlap between the blue and red arms in a very small wavelength range to connect them, that might lead to an piecing discontinuity if the signal noise ratio is too low in the overlap.

For the final spectra, spline fitting with strict flux conservation is adopted to re-bin the spectra to a common wavelength grid. Once the flux is co-added by this method, the blueand red-arms are pieced together directly and the SEDs are consistent with their target colors. For the ones which don't have photometry in the optical band, but have multiple exposures, we scaled the flux of multi-exposures to the flux of exposure with the highest SNR. After the multi-exposures being co-added, The blue and red-arm are pieced together with adjusting one of the scale (using the overlaps) to yield the final spectra.

\subsection{Accuracy analysis for flux calibration through ASPSRC}

Before discussing the accuracy of the ASPSRCs, we studied the SRCs of the DR2 plates which are derived from the LAMOST 2D pipeline to further confirm the stability of the LAMOST spectrograph response curves. Fig 5 to Fig 8 shows the distributions of the SRCs of the DR2 plates for high Galactic latitude (left panel) and for low Galactic latitude

(right panel), the standard deviations of the SRCs, as a function of wavelength, are shown by the red dashed curves.

As described in Section 2.3, we used stars with high Galactic latitude and with high SNR to get the ASPSRCs. The red solid curves in Fig 5 to Fig 8 represent the ASPSRCs, and it is consistent with the average SRC of the SRCs from the LAMOST 2D pipeline. Table 1 shows the mean uncertainties of ASPSRCs are smaller than 10\%, which are consistent with the $1 \sigma$ uncertainties of the SRCs at high Galactic latitude from the 2D pipeline. 
To verify the feasibility of applying the ASPSRCs to the flux calibration, we selected stars observed by both LAMOST and SDSS. We cross-matched the abandoned targets of LAMOST DR2 with SDSS DR12, and obtained 1,746 spectra of 1,702 stars with SNRs higher than 6 . We have calibrated the LAMOST spectra abandoned by 2D pipeline and divided them by the spectra of the same sources from SDSS. The ratios of the two spectra were calculated and then scaled to their median values of unity, and the results are shown in Fig 14. The ratios yield an average that is almost constant around 1.0 for the whole spectral wavelength coverage except for sky emission lines region; oxygen and water vapor bands of the earth's atmosphere are attributed to the uncertainties of flat-fielding and sky-subtraction. The standard deviation is less than $10 \%$ at wavelengths from $4,500 \AA$ to $8,000 \AA$ but for both edges, the standard deviation increases to $15 \%$ due to the rapid decline of the instrumental throughput. The results show that flux calibration using ASPSRCs has achieved a precision of $\sim 10 \%$ between $4,100 \AA$ and 8,900 $\AA$.

For the bright and very bright plates, most can be calibrated successfully by $2 \mathrm{D}$ pipeline. However for LAMOST faint plates (F-plates) of DR2, the flux-calibration failure rate of the $2 \mathrm{D}$ pipeline is around $9 \%$ and for the medium plates (M-plates), the failure rate is around 8\%. Fig 15 to Fig 17 show the spectra of galaxies, QSOs and stars rescued from the abandoned plates. We compared the rescued spectra with that of SDSS DR12 (the former are plotted with black curves and the latter are represented with red curves). Most match with their corresponding SDSS spectra quite well, with differences of only a few percent for their continua. For LAMOST 20130208-GAC062N26B1-sp13-112, the spectra of the red arm has turbulent components for the spectrograph No.13, this is explained by the spectrograph having problems caused by the cooling system of the CCD. For LAMOST 20140306-HD134348N172427B01-sp10-014, the SED from the ASPSRC method is bluer than that of SDSS. We believe this is due to the fact, we do not separate the Earth's atmospheric extinction from the response of the spectrograph. Generally, the variations of 
the optical atmospheric extinction curve can be calculated by low order polynomials (Patat) et al. 2011). The atmospheric extinction curve included in the ASPSRC is an average one, and multiplication by a low order polynomial is required to obtain the real atmospheric extinction curve when the target observed. Therefore, some spectra calibrated using the ASPSRCs need low order polynomials to match SDSS spectra. The atmospheric extinction of LAMOST will be deeply studied and integrated into this work.

Overall, the ASPSRCs flux calibration has achieved a precision of $\sim 10 \%$ for the LAMOST wavelength range. The potential uncertainties and temporal variations of the atmospheric extinction generally do not have an impact on the final accuracy of spectral lines, though they do affect the shapes of SEDs deduced (low order polynomials).

\section{3. $\quad$ Rescue the Abandoned Targets}

For the LAMOST DR2, there are 1,095 spectrographs with 385 plates which have been abandoned by $2 \mathrm{D}$ pipeline due to the failure of finding standard stars. We started with the 2D pipeline for fiber tracing, flux extraction, wavelength calibration, flat fielding and sky subtraction. The ASPSRCs were then adopted to calibrate the 195,694 spectra in 1,095 spectrographs. After the flux calibration and the co-add, the LAMOST 1D pipeline was employed to classify the spectra and measure the radial velocity for stars, and the redshift for galaxies and QSOs. Based on a cross-correlation method, the 1D pipeline recognizes the spectral classes and simultaneously determines the radial velocities or redshifts from the best fit correlation function. The 1D pipeline produces four primary classifications, namely STAR, GALAXY, QSO and UNKNOWN.

It is difficult to recognize galaxy and QSO spectra and determine their redshift, and as such the LAMOST 1D pipeline does not work as well as for stellar classification due to the 
SNRs of galaxy and QSO spectra being relatively lower. An additional independent pipeline, the Galaxy Recognition Module (GM for short), has been designed for galaxy recognition and redshift measurement. After the 1D pipeline was run it automatically identifies galaxies and measures the redshifts by recognizing lines. The redshifts of galaxies are determined through line centers. Before line centers are measured, a Gaussian function with sigma of 1.5 times the wavelength step is applied to the spectra to eliminate noises. The continua, which were smoothed by a median filter, are divided to complete normalization. Those data points that exceed $2 \sigma$ of a normalized spectrum are selected as candidates of emission lines, then a set of Gaussian functions is used to fit the lines. All the line centers are compared with line lists, which are spaced by steps of 0.0005 in redshift $(z)$. If most of the lines are matched successfully with heavily weighted lines such as NaD, Mgb, CaII H or CaII K for absorption galaxies, or $\mathrm{H} \alpha$, OII, $\mathrm{H} \beta$, OIII or NII for emission galaxies, the spectrum is classified as galaxy, and the corresponding $z$ is the raw redshift of the spectrum. However, for QSOs, the classifications and measurements highly depend on visual inspection. We combined the classification of GM, 1D pipeline and expert inspection and thus the final classifications of the spectra of the 1,095 spectrographs is presented in Table 2. In total 52,181 additional spectra has been recognized in DR2, and will be officially released in the Third Data Release (DR3) of the LAMOST Regular Survey. The fraction of objects rescued is about $52,000 / 2,000,000(\sim 2.5 \%)$.

For the rescued 52,181 targets, we evaluated the quality by plotting the magnitude against SNR relationships for galaxies and QSOs, and stars. For galaxies and QSOs, most of the magnitudes are spread between 17.0 and 19.0, which is shown in Fig 18. This is close to the limit of LAMOST observation, consequently, the majority of their SNRs are so low that they do not reach a SNR of 10. To reduce the differences of SNRs due to differences in exposure times, all of the SNRs in this paper were scaled to 5400s. For stars, there are two peaks in the distribution of magnitudes, as shown in Fig 19 . The 
magnitudes of A,F,G,K-type stars range from 13.0 to 17.0, and M-type stars range from 15.0 to 18.0 magnitudes. The SNRs of the stars are higher than that of galaxies and QSOs, however, most are below 30, which is comparatively low for stars. With the exception of M-type stars, we selected the stars with SNRs in the $r$ band larger than 2.0 for the release. Therefore, an obvious cut is seen in the bi-modal point distributions of early and late type stars in Fig 19, For F,G,K-type stars, by running LASP, we parameterized those with SNRs in the g band larger than 6.0 for nights with a dark moon and 15.0 for nights with a bright moon. The final stellar parameters coverage is presented in Fig 20.

\subsection{Revision of the $2 \mathrm{D}$ calibration}

To minimize potential errors introduced by poor sky subtraction, the current LAMOST 2D pipeline (v2.7) scales the sky spectrum to obtain the same flux intensities of sky emission lines as that of the target spectra, which it will be subtracted from. It is assumed that the emission lines are homogeneous across the FoV of individual spectrographs (about 1

deg). However, the continuum sky-background and sky emission lines originate from very different sources and are excited by different mechanisms, thus their emission levels are unlikely to scale linearly. In fact, even amongst the sky emission lines, lines from different species may have quite different behavior in terms of their temporal and spatial variations Oliva et al. 2015). Consequently, scaling the sky spectra by the measured fluxes of sky emission lines risks subtracting an incorrect level of sky background. For a minority of spectra the standard stars telluric bands are extremely under subtracted, and thus it turns out that the SRCs of the standards are over-fitted (see Fig 21). The oxygen band is under-subtracted for the spectra of the standards. This leads to the over fitted SRC also containing the oxygen band and introduces artificial spectral lines to all the spectra of the spectrograph, making the classification of spectra by 1D pipeline difficult. 
Fig 22 shows one example of an artificial spectra calibrated by the over-fitted SRC (from Fig 21 using the 2D pipeline), which is plotted with black curves. We recalibrated the spectra using the ASPSRCs, which is presented with red curves in Fig 22. After recalibration, the spectra was classified as F0 by the 1D pipeline (an improvement over the 'Unknown' classification by the 1D pipeline previously). Comparing the ASPSRCs with the SRCs from the LAMOST 2D pipeline, we found 6 spectrographs from DR2 have this problem, and all of the 6 plates were observed in the nights with very bright moon. The ASPSRC method has been used to correct this problem, and the spectra of this 6 spectrographs will be released in LAMOST DR3.

\section{Analysis and Discussions}

We have applied the ASPSRCs to the flux calibration for LAMOST, however, there are still some uncertainties in the ASPSRCs caused by individual SRCs. The causes of these variations in the shape of SRCs might be attributed to several factors.

First of all, although we selected the standard stars from high galactic latitudes to minimize the affects from variations of interstellar extinction, the effect of the Earth's atmospheric extinction still exist. Typical atmospheric extinction curves are smooth

functions of wavelength in the LAMOST wavelength coverage (Bongard et al. 2013; Cullen et al. 2011), and this is usually true for the variations of atmospheric extinction, which can be well represented by low order polynomials. Therefore, the mean atmospheric extinction curve included in the ASPSRCs does not affect the spectral lines of the calibrated spectra. Its variations are included in the overall variations of SRCs.

Secondly, fiber positioning may introduce variations in the fiber spectral response during the tracing of the targets. This means the SRCs of the individual fibers probably 
vary from observations of one plate to another (Chen et al. 2015). The variations of the fiber flat fields will have an impact on sky subtraction and flux calibration, introducing the uncertainties to the SRCs. To make matters worse uncertainties introduced by such variations do not depend on the spectral SNRs. That is to say, even spectra of very high SNRs may have incorrectly shaped SEDs. Attempts to characterize and correct for such variations of the fiber flat fields is under way. If the condition allows, it is better to obtain the ASPSRCs once a year or once a quarter to overcome these instrumental changes.

Thirdly, the spectral SNRs of standard stars have an impact on the SRCs derived from them. To test how the SEDs of LAMOST Galactic targets are affected by limited SNRs, the spectral and photometric $(\mathrm{g}-\mathrm{r})$ colors have been compared as a function of the spectral SNRs. The results shows that at SNRs exceeding 20, the spectral colors and photometric colors agree well, with a mean difference of 0.01-0.02 mag and no systematic trend, while at SNRs lower than about 10, the discrepancies increase rapidly, along with some systematic differences (Xiang et al. 2015). To minimize the uncertainties introduced by spectral SNRs, we selected the standard stars with SNRs larger than 20 to obtain the ASPSRCs.

In addition, errors due to the stellar atmospheric parameters of standard stars also cause variations in the SRCs. For flux standard stars of $5,750 \mathrm{~K} \leq T_{\text {eff }} \leq 6,750 \mathrm{~K}$, an error of $150 \mathrm{~K}$ in $T_{\text {eff }}$ can lead to a maximum uncertainty of $12 \%$ in the shape of the stellar SED and thus it will change the shape of the SRC derived from it. Uncertainties caused by errors in $\log \mathbf{g}$ are negligible (i.e. for an estimated uncertainty of $0.25 \mathrm{dex}$ in $\log \mathrm{g}$, about $1 \%$ for the whole wavelength range is affected). Metallicity mainly affects the blue-arm spectra at wavelengths less than $4,500 \AA$. An error of 0.2 dex in $[\mathrm{Fe} / \mathrm{H}]$ can change the SED shape between 3,800 $\AA$ and 4,500 $\AA$ by approximately $3 \%$, while the effects at wavelengths greater than 4, $500 \AA$ are only marginally (Xiang et al. 2015). This is the reason we remove the candidates with standards which above uncertainties in $T_{\text {eff }}$ larger than $150 \mathrm{~K}$. 
The advantage of the ASPSRCs comes from using an average SRC of instrument response curves and the average of atmospheric extinction curves, although there are many uncertainties introduced by the influencing factors discussed, which will eliminate the effects. Our experiments prove that all the influencing factors on accuracy of flux calibration is less than 10 during the DR2 period. The average SRCs are presented in Table 3 to Table 6. One can use them to calibrate spectra of the LAMOST DR2 catalogue. For the spectra observed subsequent to DR2, new ASPSRCs will need to be produced to counter variations from the instrument.

\section{Acknowledgements}

The authors would like to thank M.-S. Xiang, H.-B. Yuan for helpful discussion. This work is supported by the by the National Key Basic Research Program of China (Grant No.2014CB845700), and National Science Foundation of China (Grant Nos 11390371, 11233004). Guoshoujing Telescope (the Large Sky Area Multi-Object Fiber Spectroscopic Telescope, LAMOST) is a National Major Scientific Project built by the Chinese Academy of Sciences. Funding for the project has been provided by the National Development and Reform Commission. LAMOST is operated and managed by the National Astronomical Observatories, Chinese Academy of Sciences. 


\section{REFERENCES}

A-Li Luo, et al., 2015, RAA, 15, 8, 1095-1124

Bongard, S., Canto, A., Cellier-Holzem, F., et al., 2013, A\&A, 549, 8

Castelli, F.,Kurucz, R. L., et al., 2004, New Grids of ATLAS9 Model Atmospheres, arXiv, 0405087

Chen, J.J., Bai, Z. R., et al., 2015, RAA, 15, 608

Cui, X.Q., Zhao, Y. H., et al., 2012, RAA, 12, 1197

Cullen, H. B., Margaret M. S., PASP, 123, 1302

Deng, L.C., Newberg, H. J., Liu, C., et al., 2012, RAA, 12, 735

Patat, F., Moehler, S., et al., 2011, A\&A , 527, 91

Fitzpatrick, E. L. , et al., 1999, PASP, 111, 63

Fitzpatrick, E. L., Massa, D., 2007, ApJ, 663, 320

Hamuy, M., Walker, A. R., Suntzeff, N. B., et al., 1992, PASP, 104, 533

Hamuy, M., Suntzeff, N. B., Heathcote, S. R., et al., 1994, PASP, 106, 566

Grupp, F., 2004, A\&A, 426, 309

Oke, J. B., Gunn, J. E., 1983, AJ, 266, 713

Oke, J. B., et al., 1990, AJ, 99, 1621

Oliva, E., Origlia, L., Scuderi, S., et al., 2015, A\&A, 581, 47

Pickles, A., 2010, UBVRI-ZY and ugriz zeropoints from 19 calspec standards, STSCI 
Schlafly, E. F., Finkbeiner, D. P., Schlegel, D. J., 2010, ApJ, 725, 1175

Schlegel, D. J., Finkbeiner, D. P., Davis, M., 1998, ApJ, 500, 525

Song, Y.H., Luo, A.-L., Comte, G. et al., 2012, RAA, 12, 453

Stoughton, C., Lupton, R. H., Bernardi, M. et al., 2002, AJ, 123, 485

Tonry, J. L., Stubbs, C. W., Lykke, K. R. et al., 2012, AJ, 750, 99

Xiang, M.X., Liu, X.W., et al., 2015, MNRAS, 448,90,103

Wu, Y., et al., 2011, RAA, 11, 8, 924-946

Wu, Y., et al., 2011, AA, 525, A71

Yanny, B., Rockosi, C., Newberg, H. J. et al., 2009, AJ, 137, 4377

York, D. G., Adelman, J., Anderson, J. E. et al., 2000, AJ, 120, 1579 


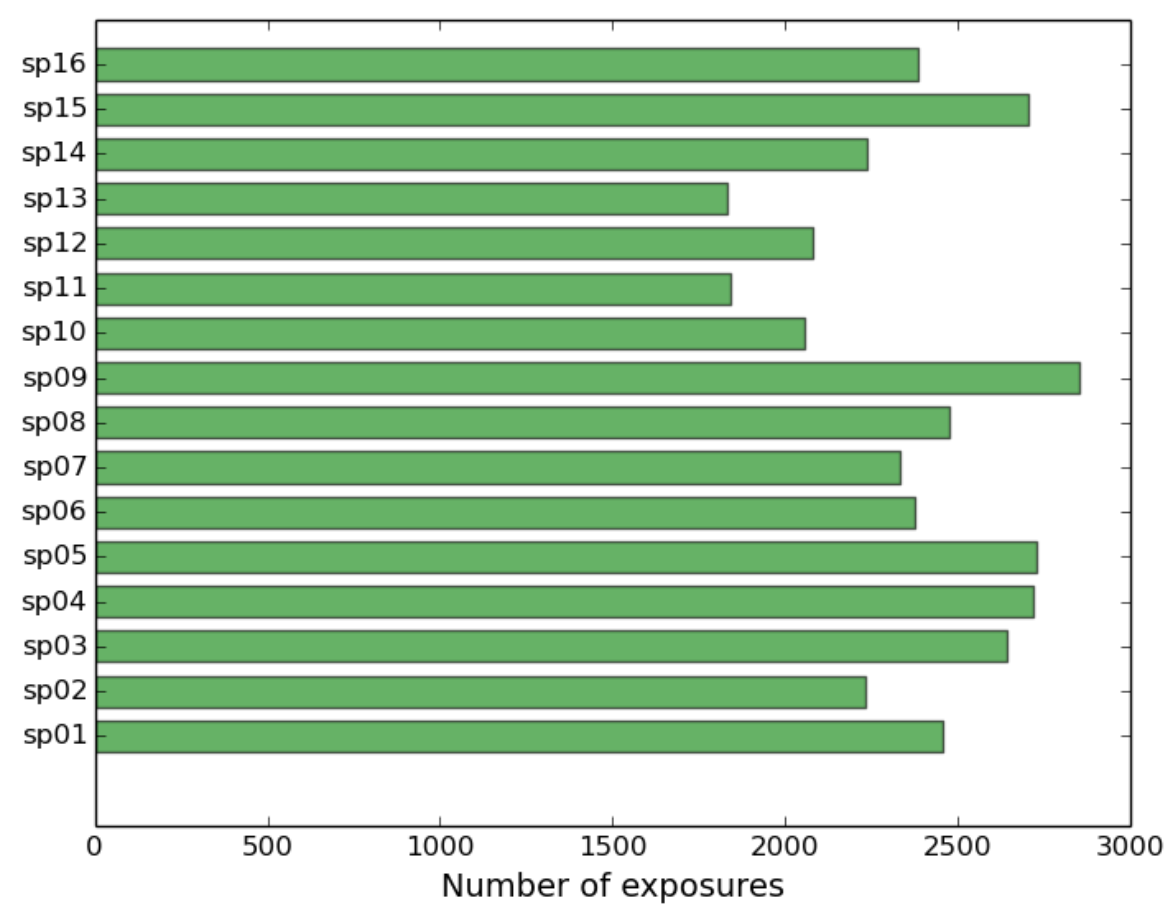

Fig. 1.- Histogram of the numbers of exposures of standard stars selected per spectrograph. 


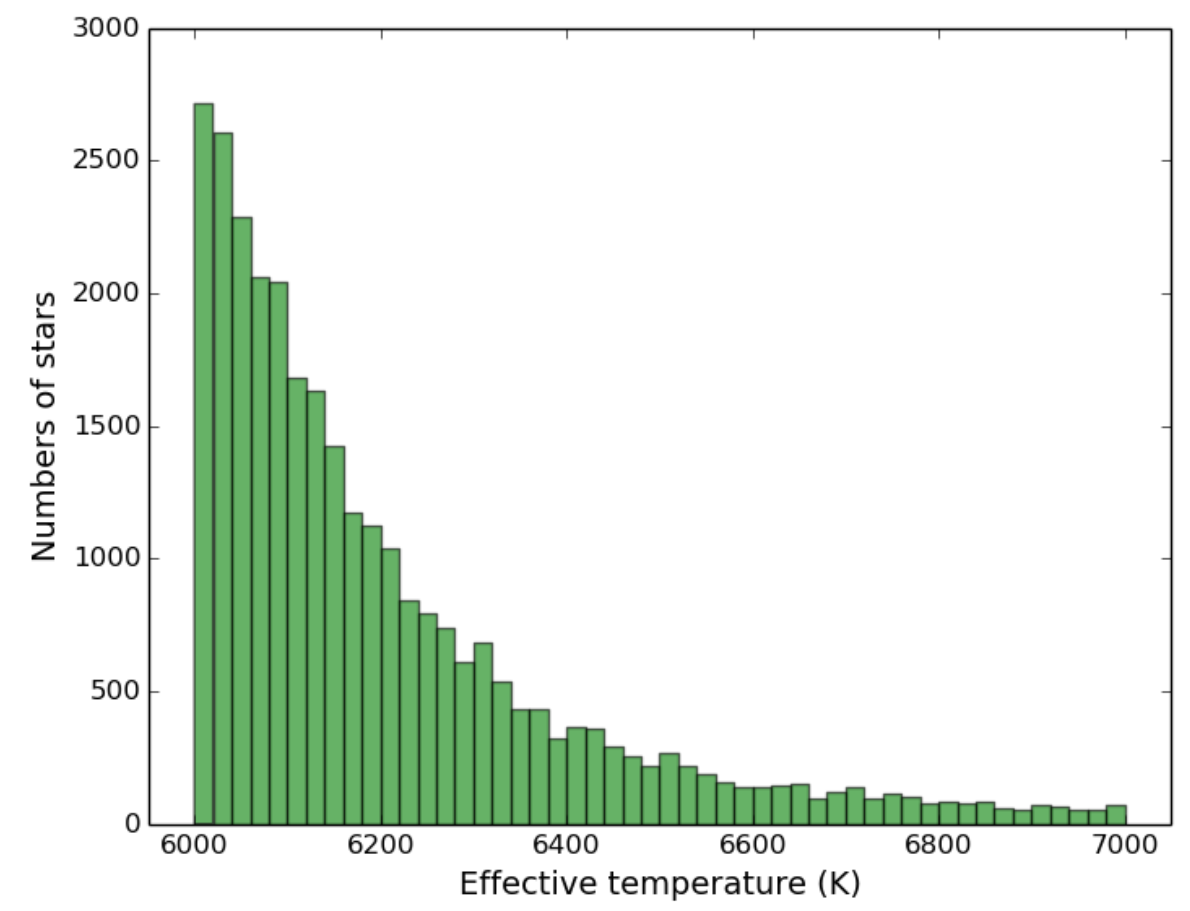

Fig. 2.- Histogram of the effective temperatures of the selected standard stars. 

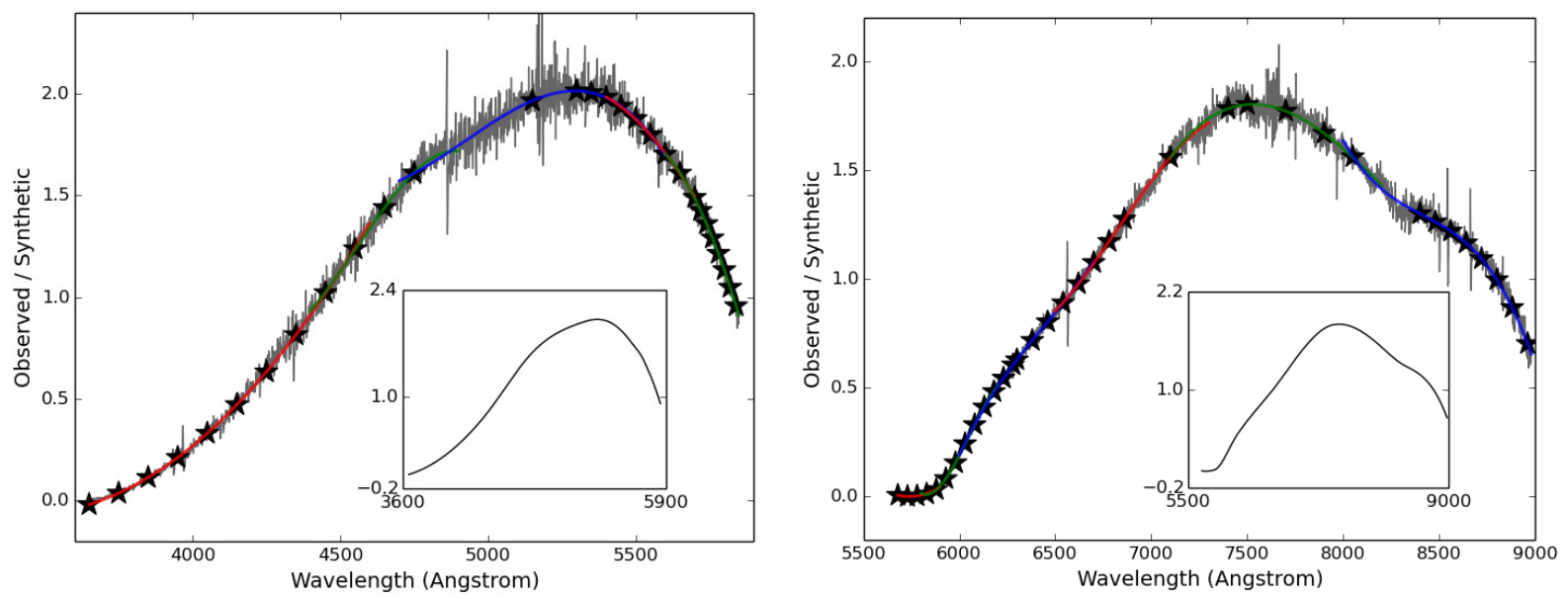

Fig. 3.- Examples of SRC fitting for blue- (left) and red-arm (right) spectra. The grey lines are the ratios of observed flux density and the synthetic flux density, the ratios have been scaled by their mean value. The blue- and red-arm spectra are divided into five and six wavelength regions respectively, and each region is fitted with a second- or third-order polynomial, which are represented by the thick lines in RGB colors (e.g. The blue arm has 5 bins drawn in the order of red, green, blue, red and green; while the red arm has six bins drawn in the order of red, green, blue, red, green, and blue). Asterisks are selected from the fitted polynomial curves avoiding prominent spectral features, which are used for the final SRC fitting. 

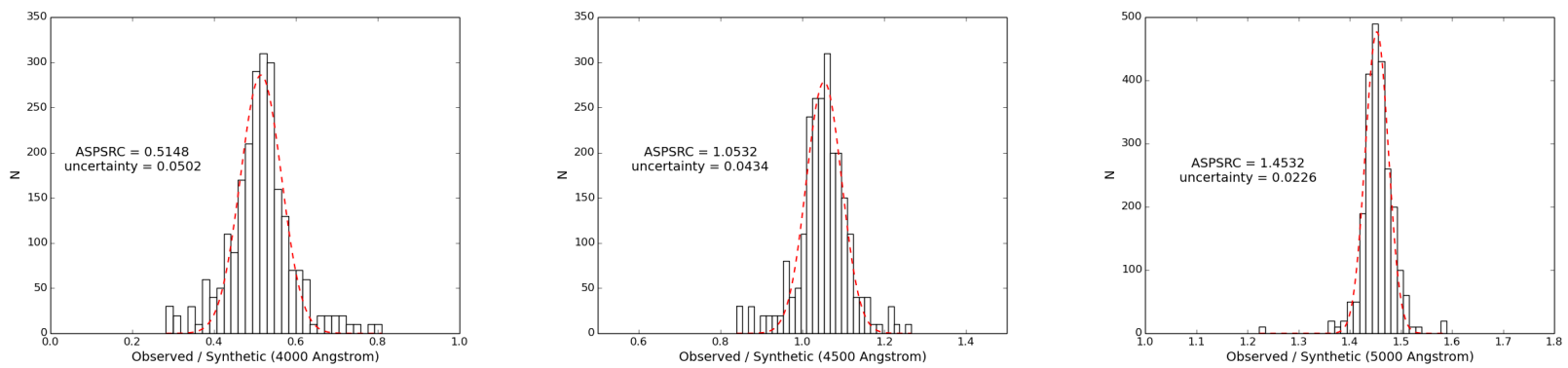

Fig. 4.- Histograms of SRCs in 4,000 (left), 4,500 $\AA$ (middle) and 5,000 $\AA$ (right) of spectrograph No.1, the red dashed curves are Gaussian fits to the distributions, the mean and dispersion of the Gaussian fit to the ASPSRC and uncertainty are also marked. 


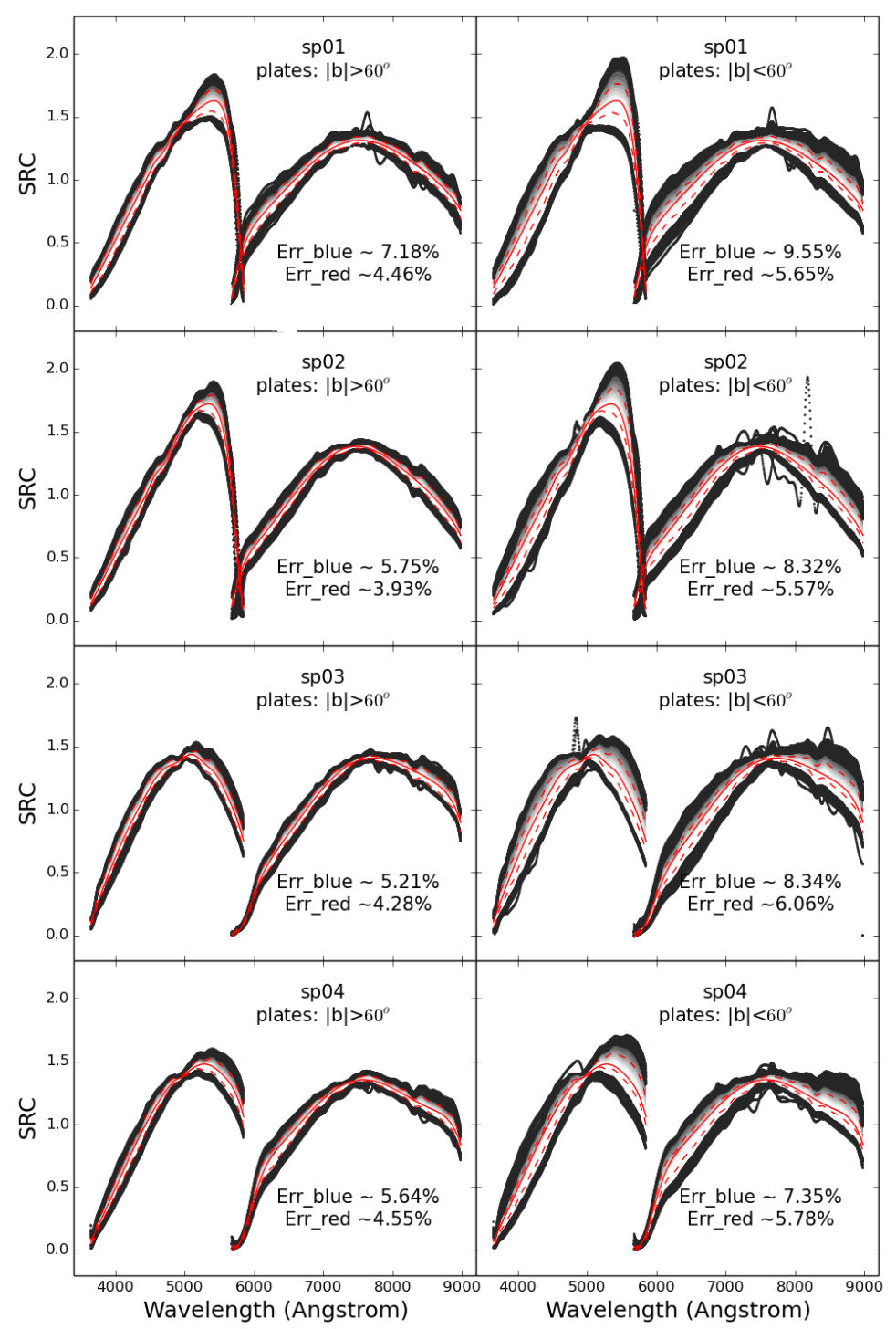

Fig. 5.- The distributions of SRCs of spectrographs from No.1 to No.4, the SRCs were derived from 2D pipeline for the DR2 plates. The grey contours represent the distributions of SRCs from 372 plates with 914 exposures for high galactic latitude in left panel, and the distributions of SRCs from 1,759 plates with 3,387 exposures for the low galactic latitude in right panel. The standard deviation of the SRCs as a function of wavelength is shown by the dashed curves, and the ASPSRCs we described in this paper are shown by solid curves. 


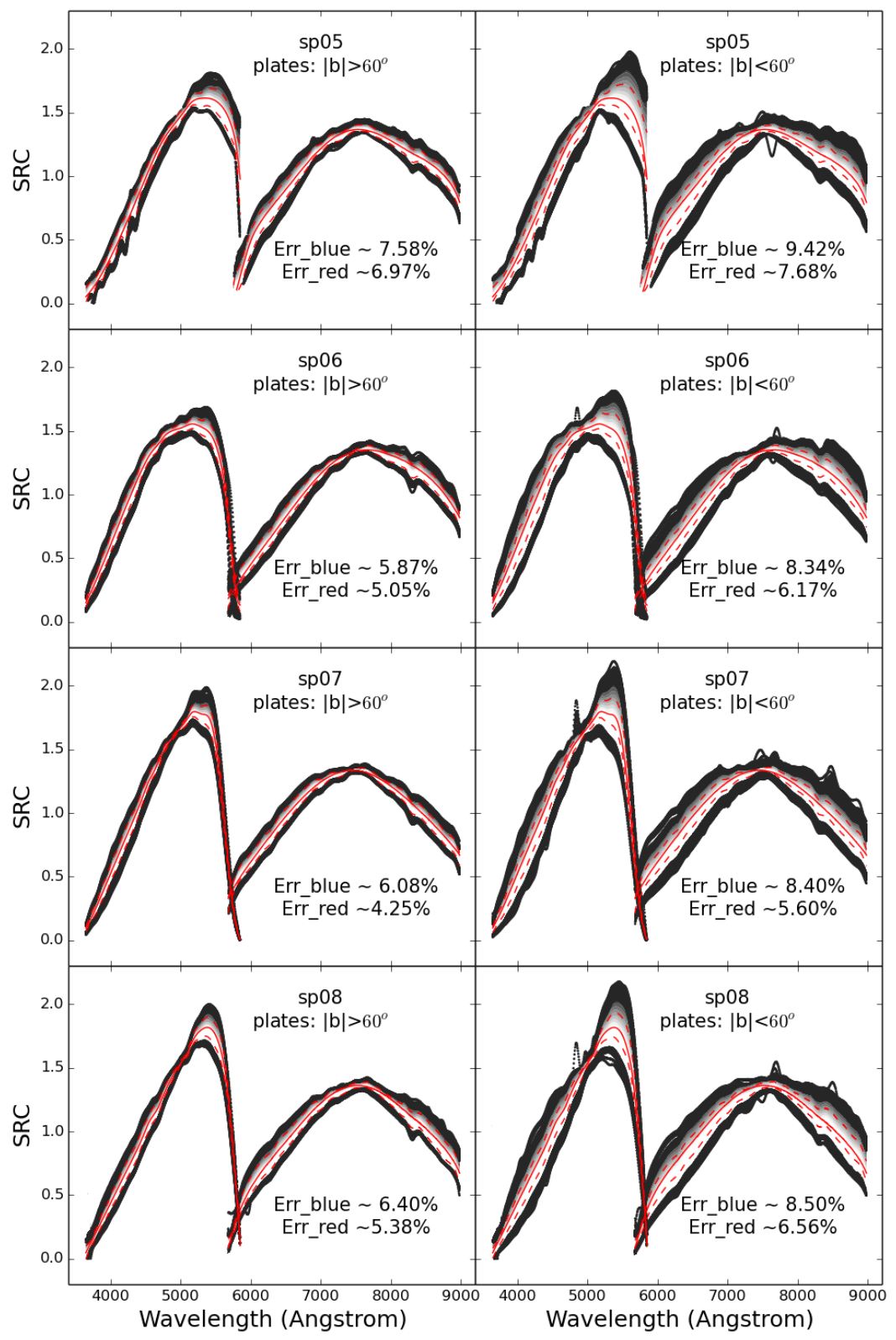

Fig. 6.- The distributions of SRCs of spectrographs from No.5 to No.8. The convention is the same as in Fig 5. 


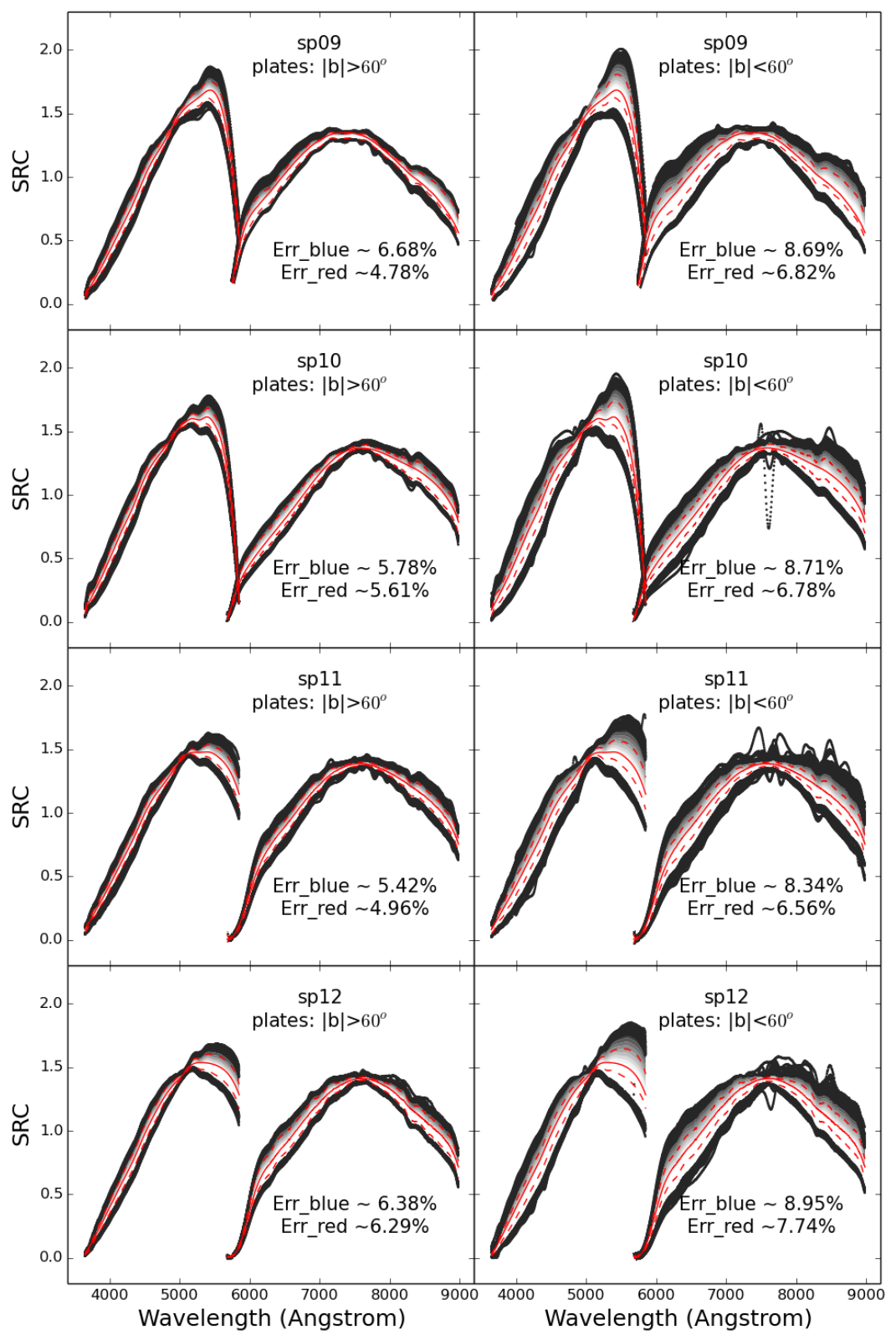

Fig. 7.- The distributions of SRCs of spectrographs from No.9 to No.12. The convention is the same as in Fig 5 . 


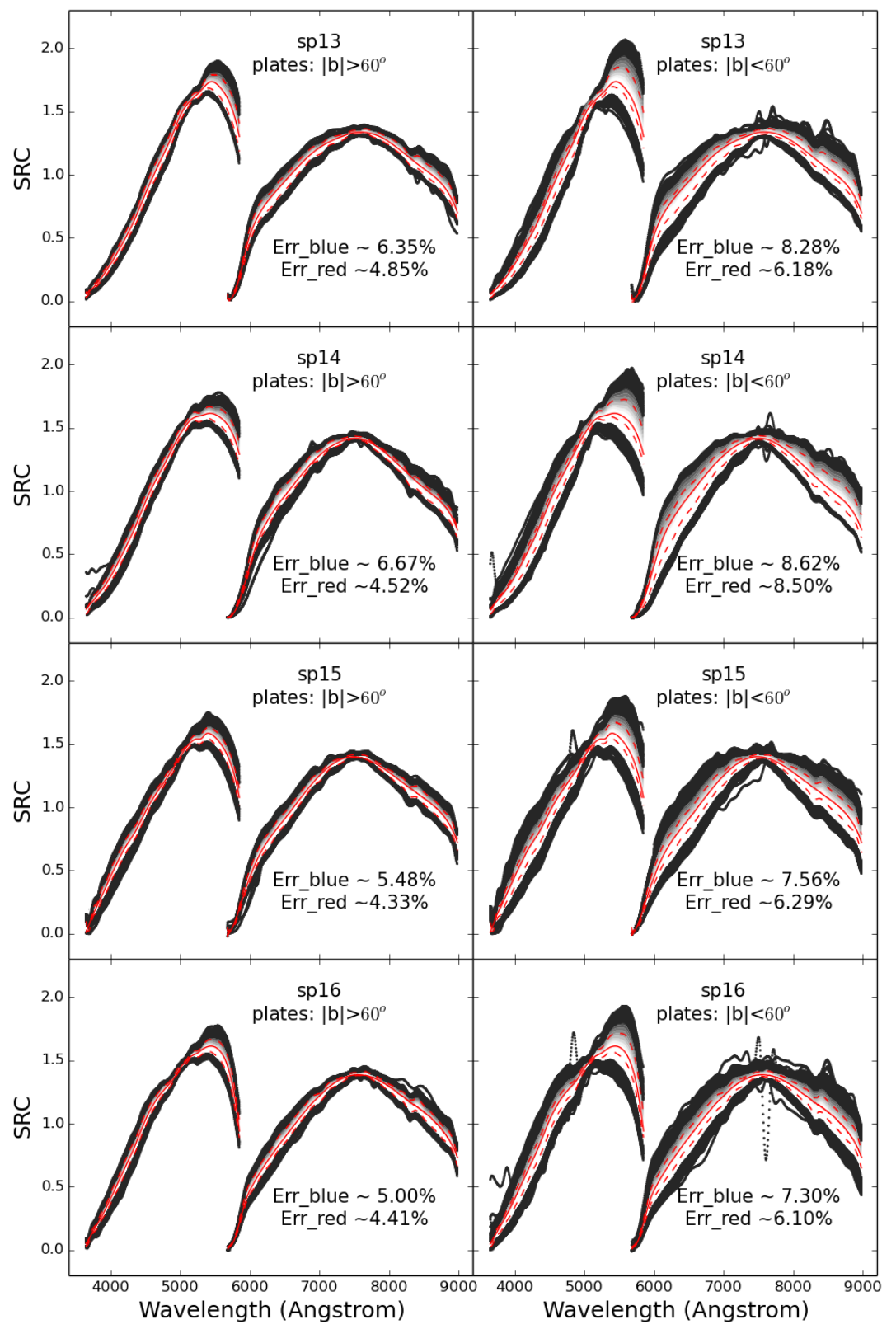

Fig. 8.- The distributions of SRCs of spectrographs from No.13 to No.16. The convention is the same as in Fig 5 . 


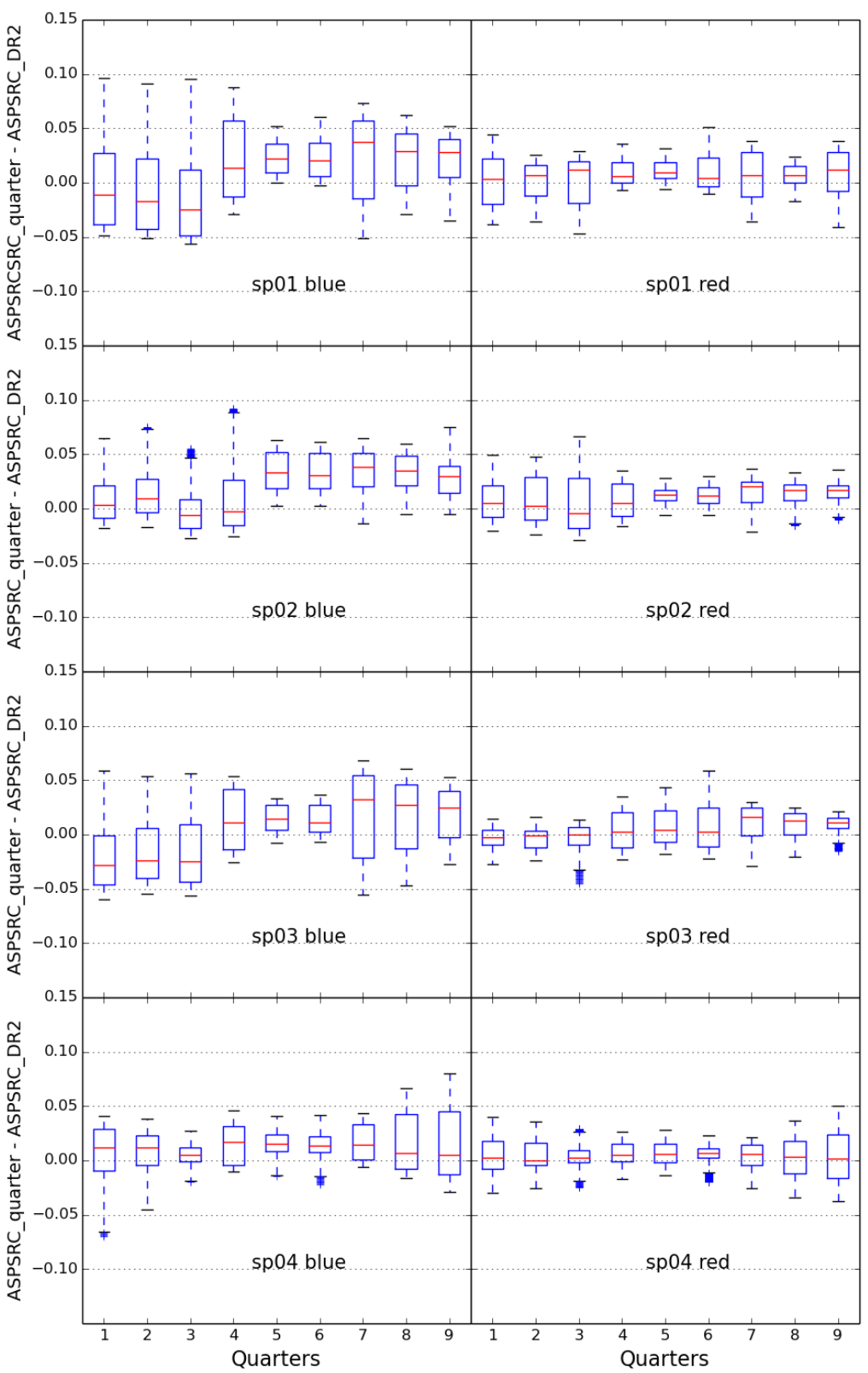

Fig. 9.- The distributions of residuals between the nine Quarter ASPSRCs and the DR2 ASPSRC (blue arm in left panel and red arm in right panel), for spectrographs from No.1 to No.4. The box extends from the lower to upper quartile values of the error, with a line at the median. The whiskers extend from the box to show the range of the error. Flier points are those past the end of the whiskers. 


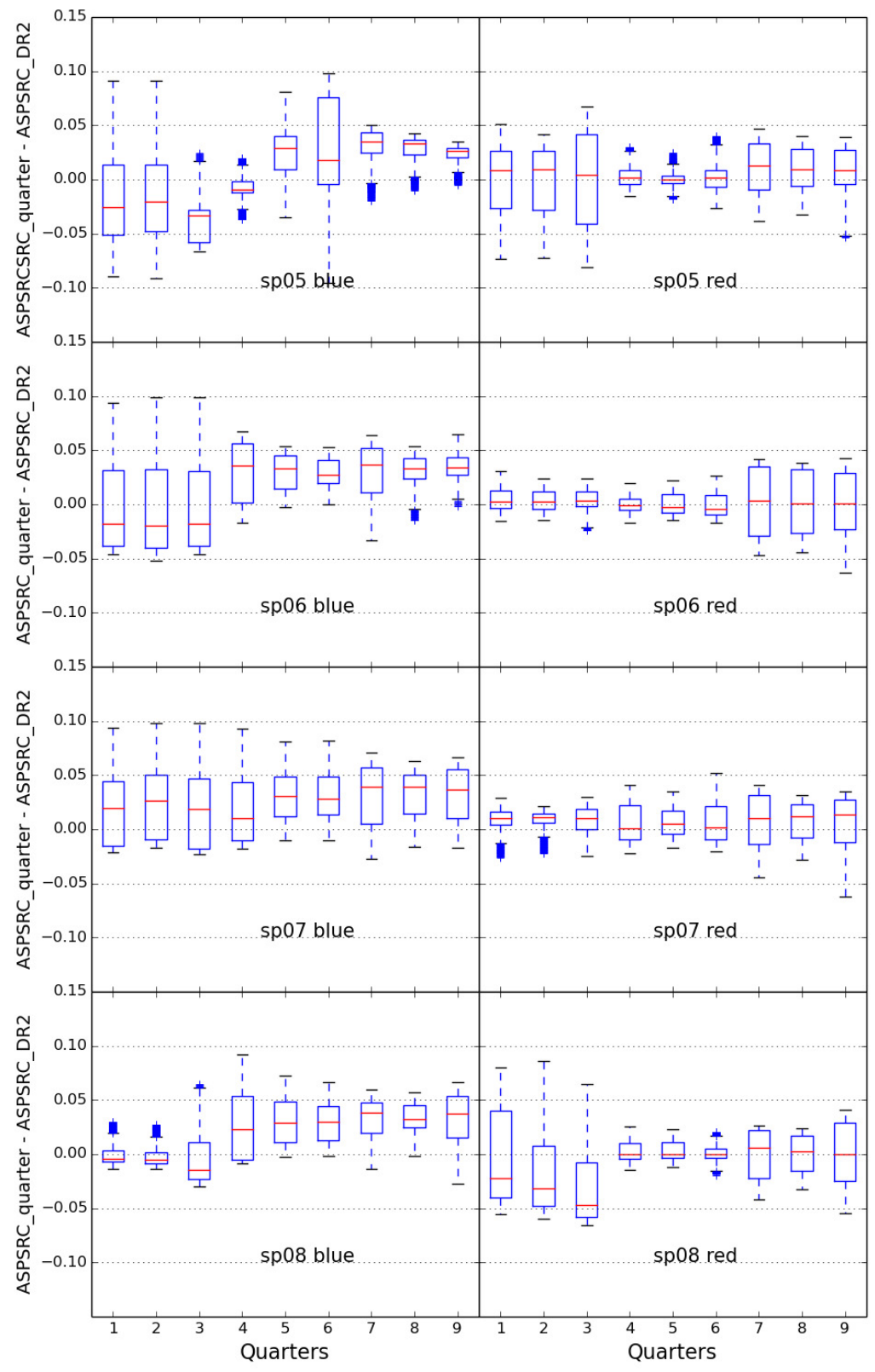

Fig. 10.- The distributions of errors for spectrographs from No.5 to No.8. The convention is the same as in Fig 9 . 


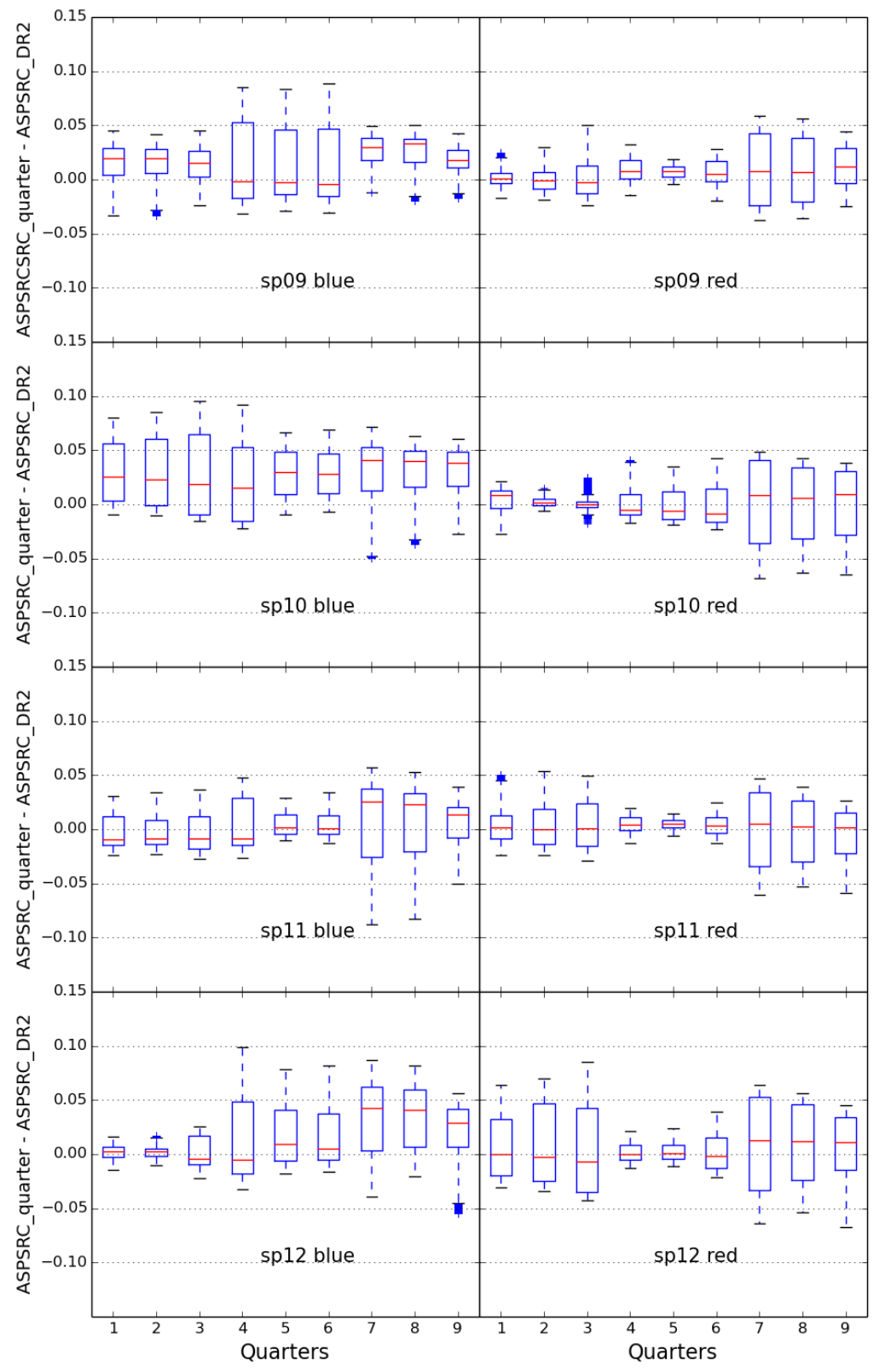

Fig. 11. - The distributions of errors for spectrographs from No.9 to No.12. The convention is the same as in Fig 9 . 


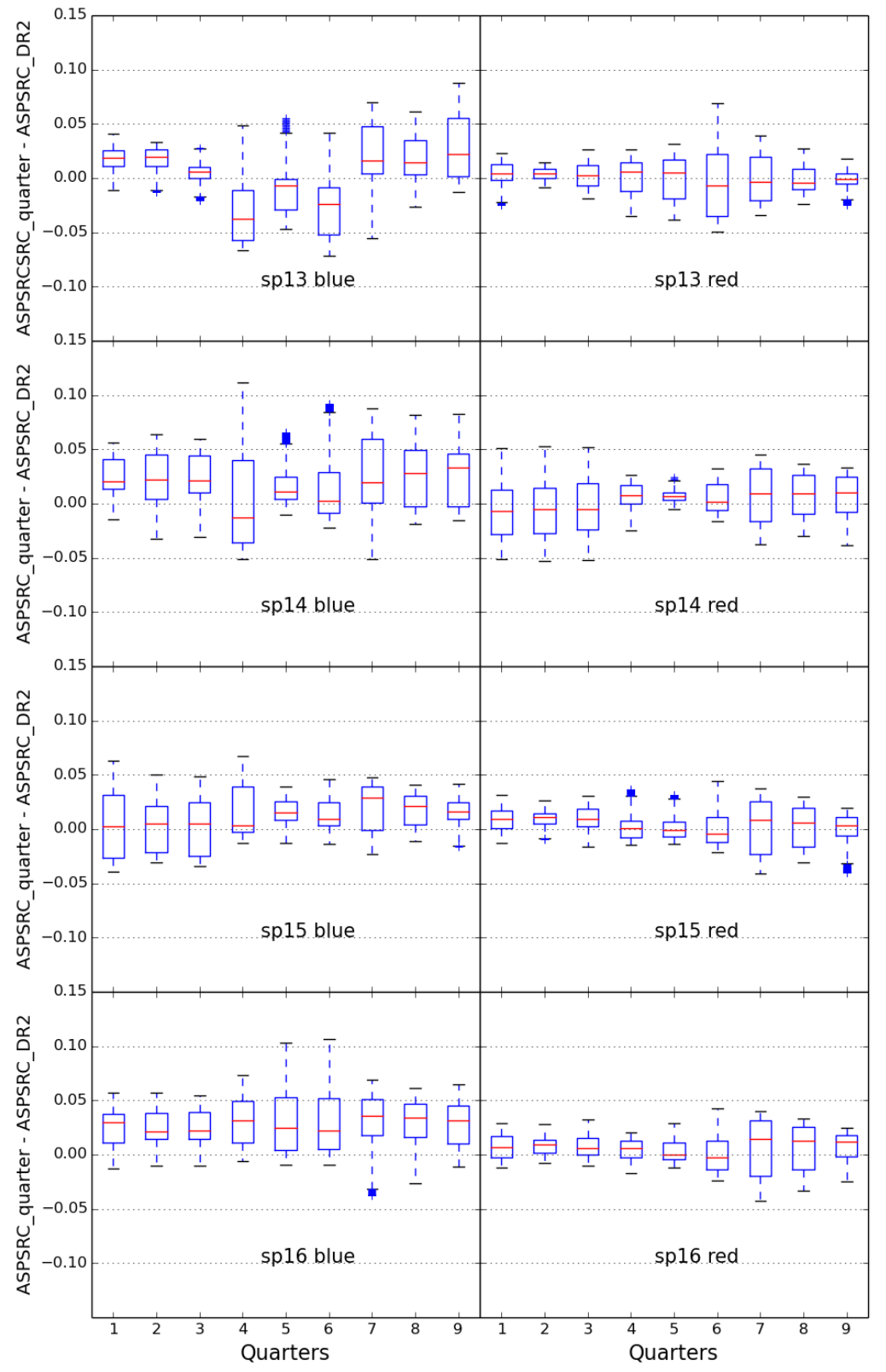

Fig. 12.- The distributions of errors for spectrographs from No.13 to No.16. The convention is the same as in Fig 9 . 


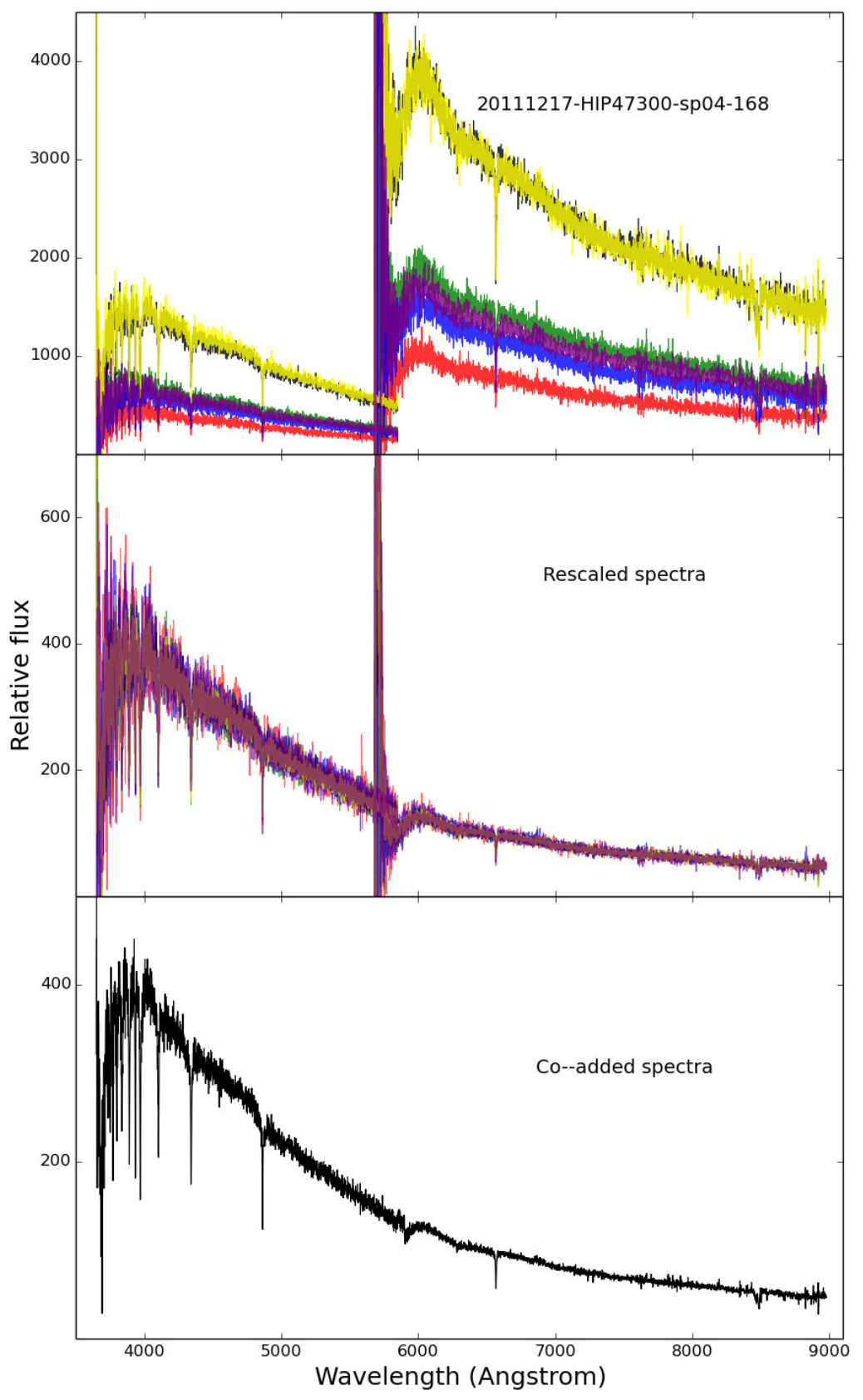

Fig. 13.- Example of a star with 6 exposures in different scales, for blue and red-arms, the spectra of equal exposure are plotted by the same color (top). The rescaled spectra (middle) are scaled depend on $\mathrm{g}$ and i magnitudes. The co-added spectra (bottom) are adopted as the final spectra. 


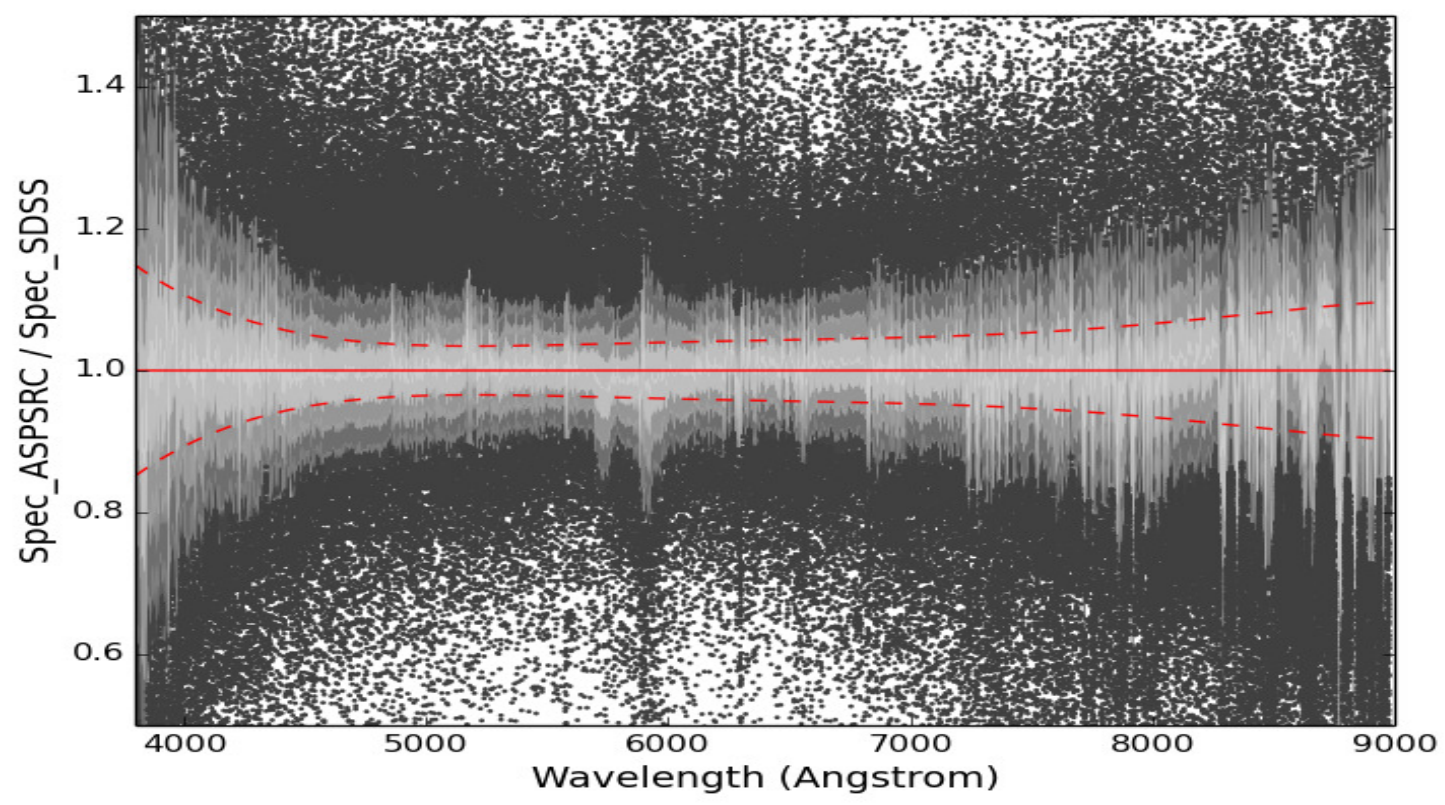

Fig. 14.- Distribution of the ratios of spectral pairs observed by both LAMOST and SDSS, each point on the panel is a ratio value of one pair, the contour represent the distribution of the ratio values of 1,746 spectral pairs. The smoothed mean and standard deviation of the ratios, as a function of wavelength, are shown by the solid and dashed curves. 


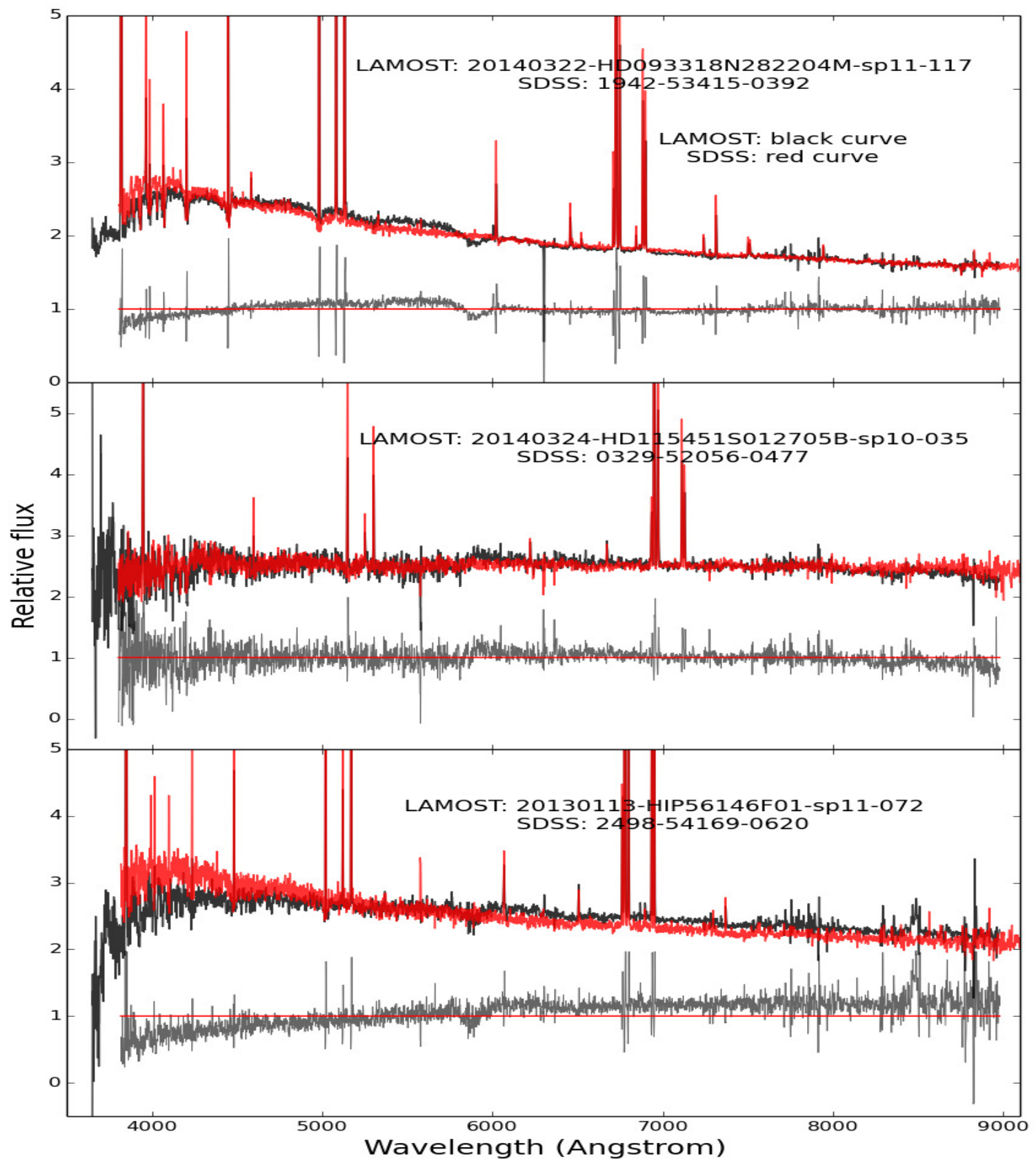

Fig. 15. - Comparison of the rescued spectra of galaxies (black) with SDSS DR12 spectra (red). For each panel, the upper part shows the relative flux density as a function of wavelength, whereas the lower part shows the ratios of LAMOST and SDSS. 


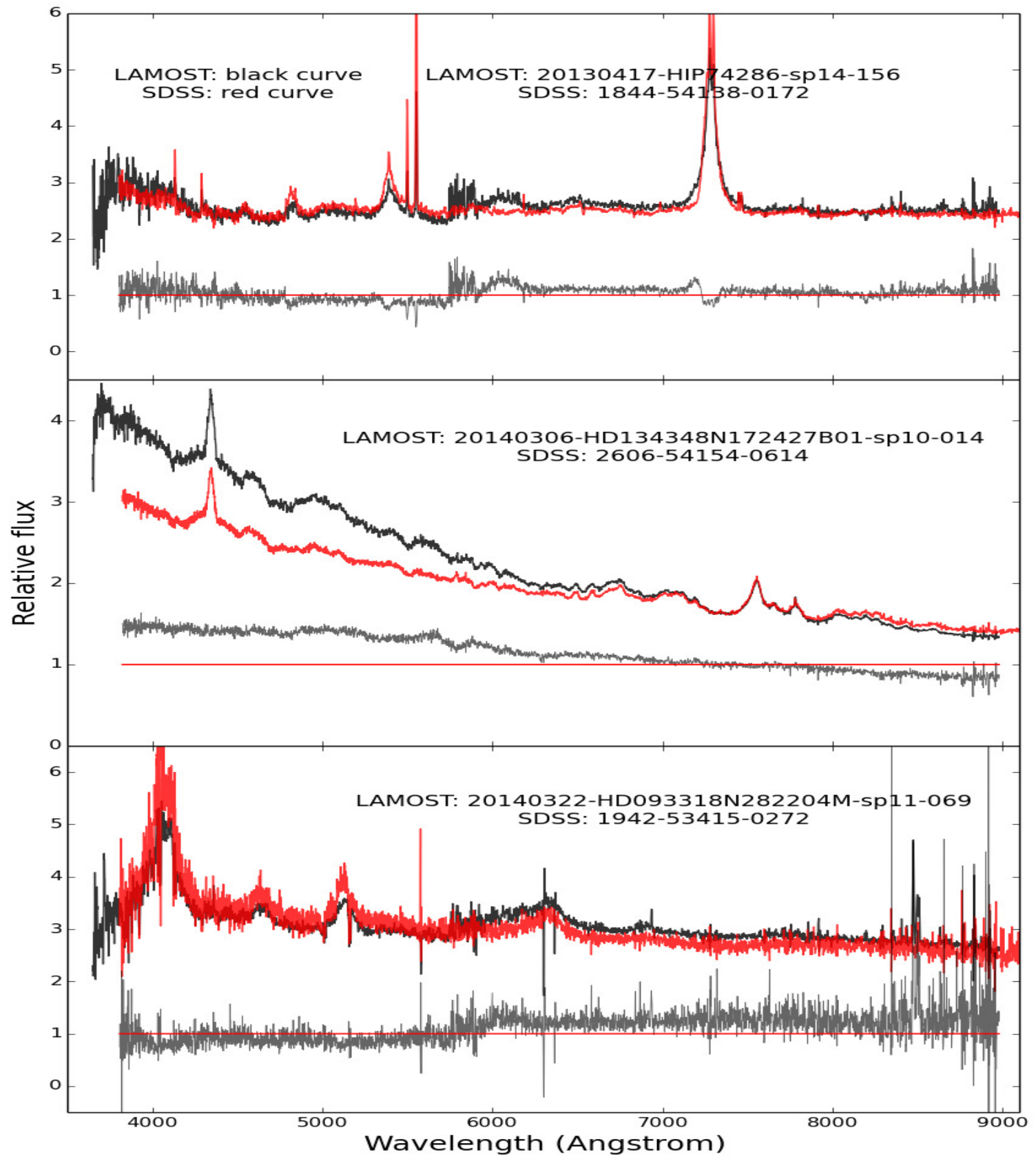

Fig. 16. - Comparison of the rescued spectra of QSOs (black) with SDSS DR12 spectra (red). 


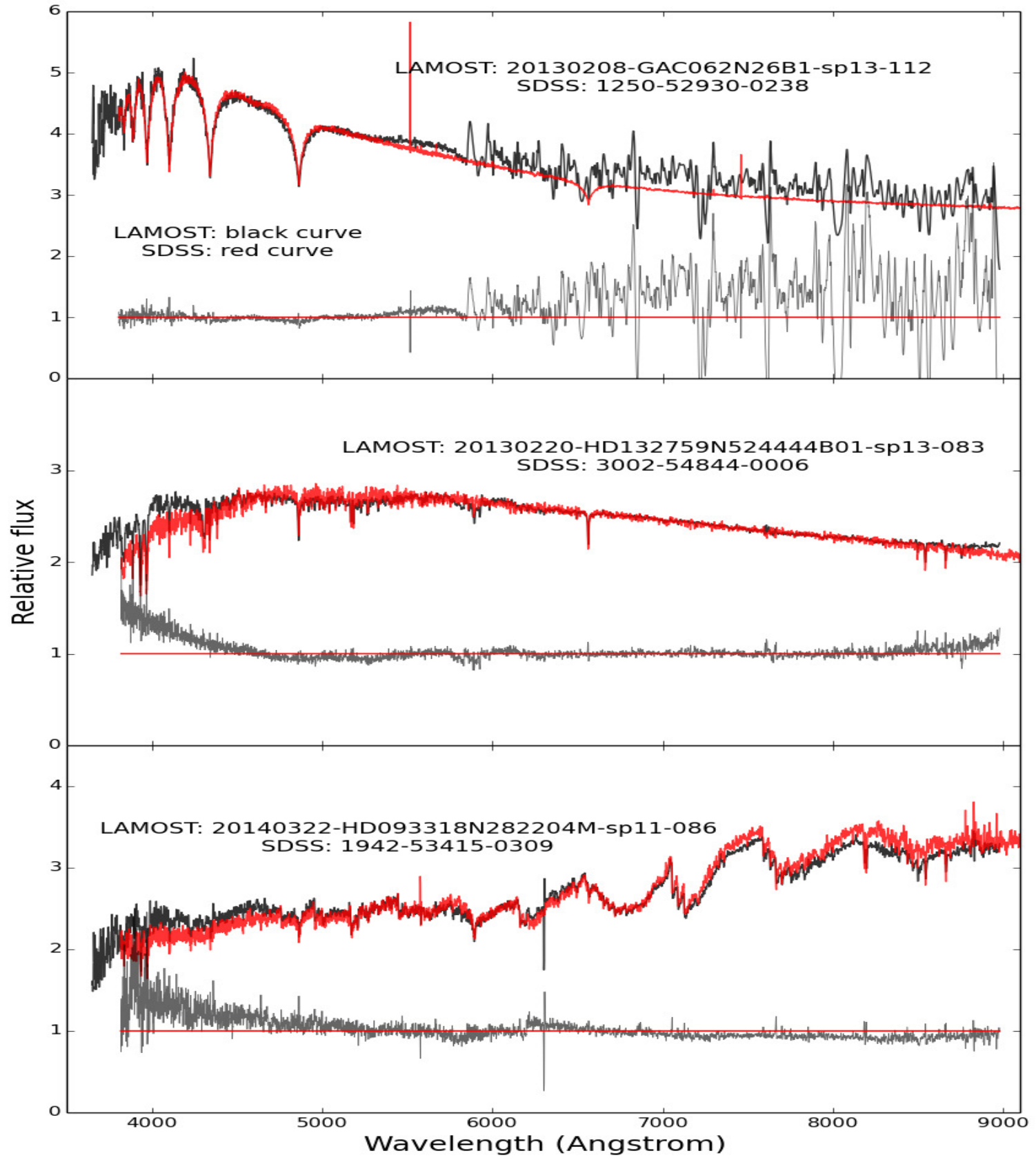

Fig. 17.- Comparison of the rescued spectra of stars (black) with SDSS DR12 spectra (red). 


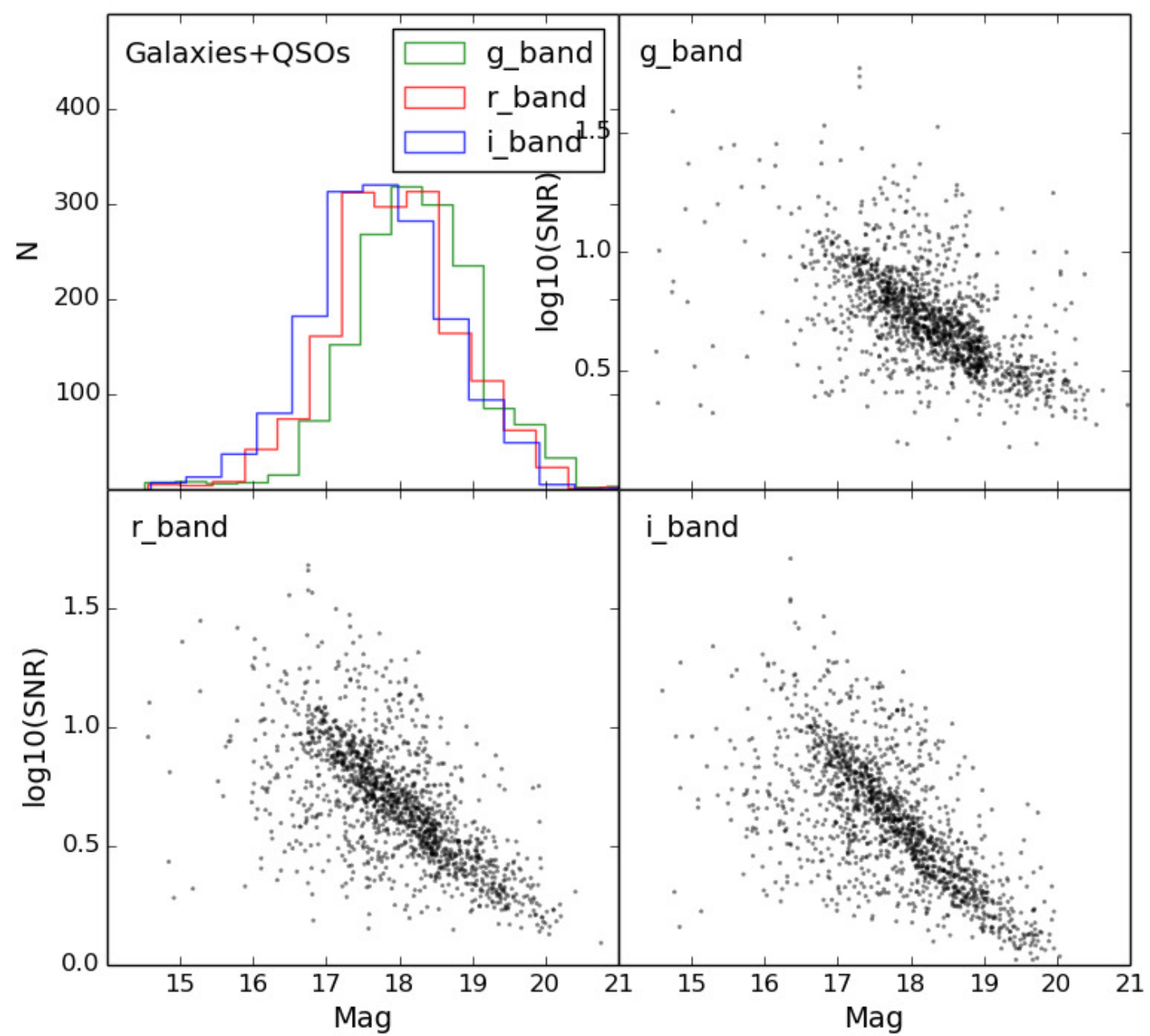

Fig. 18. - Histogram of the g,r,i magnitudes of galaxies and QSOs, which are rescued by the ASPSRCs from the abandoned spectrographs of the 2D pipeline. The diagrams of SNRs and magnitudes are provided for $\mathrm{g}, \mathrm{r}, \mathrm{i}-\mathrm{bands}$. 


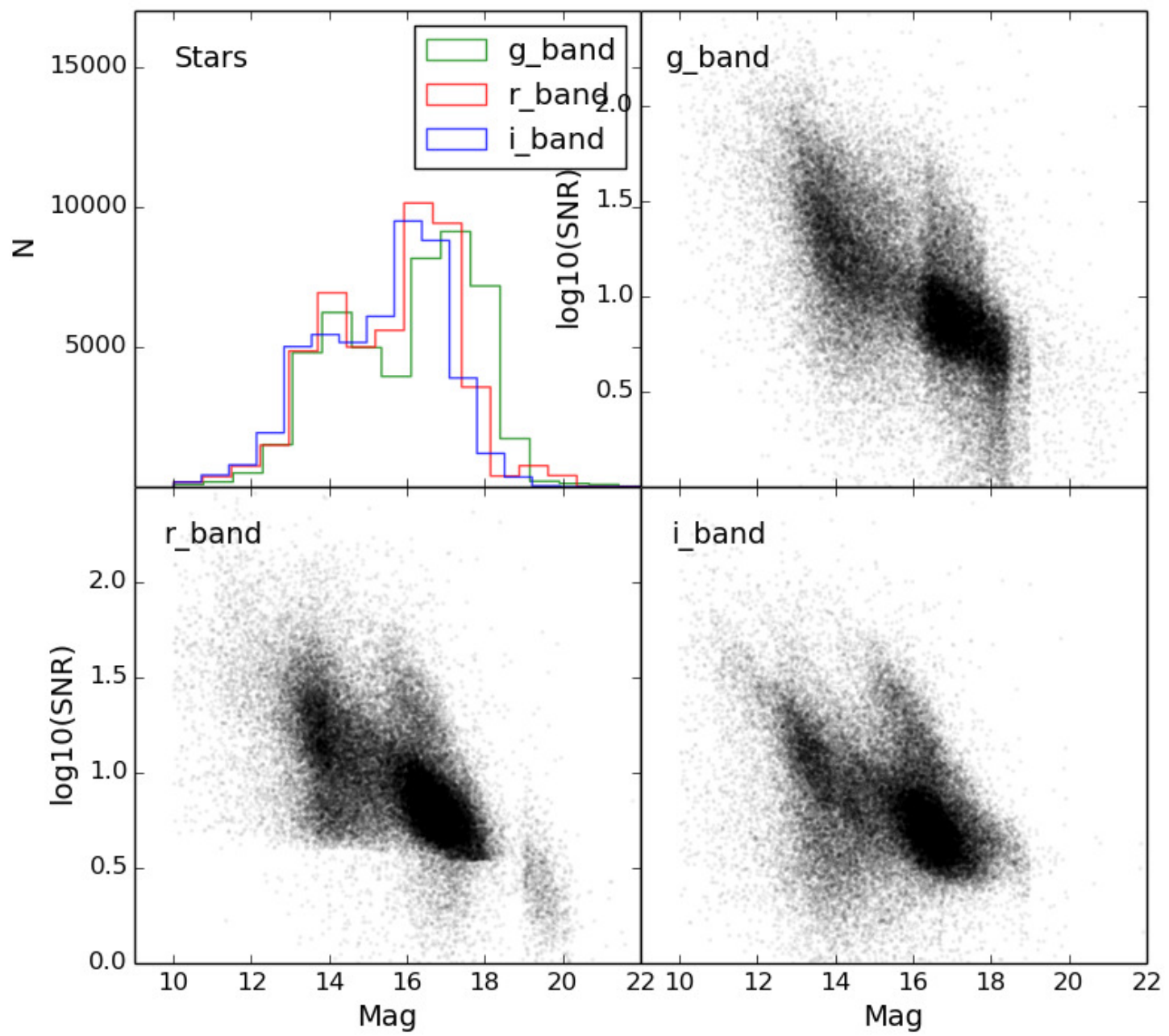

Fig. 19. - Histogram of the g,r,i magnitudes of stars, which are rescued by the ASPSRCs from the abandoned spectrographs of the 2D pipeline. The diagrams of SNRs and magnitudes are provided for $\mathrm{g}, \mathrm{r}, \mathrm{i}-\mathrm{bands}$. 


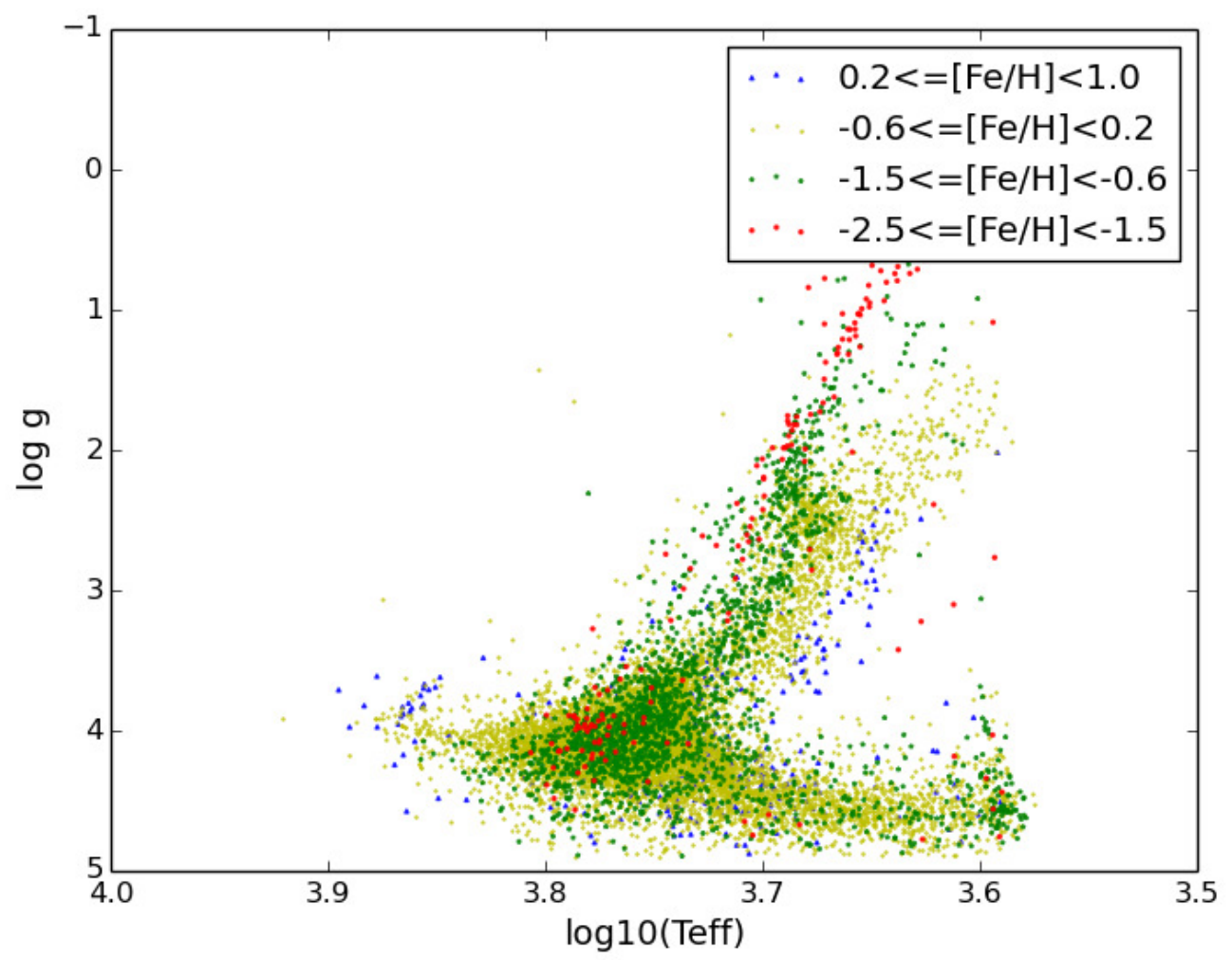

Fig. 20. - The coverage map of stellar parameters of F,G,K-type stars. 


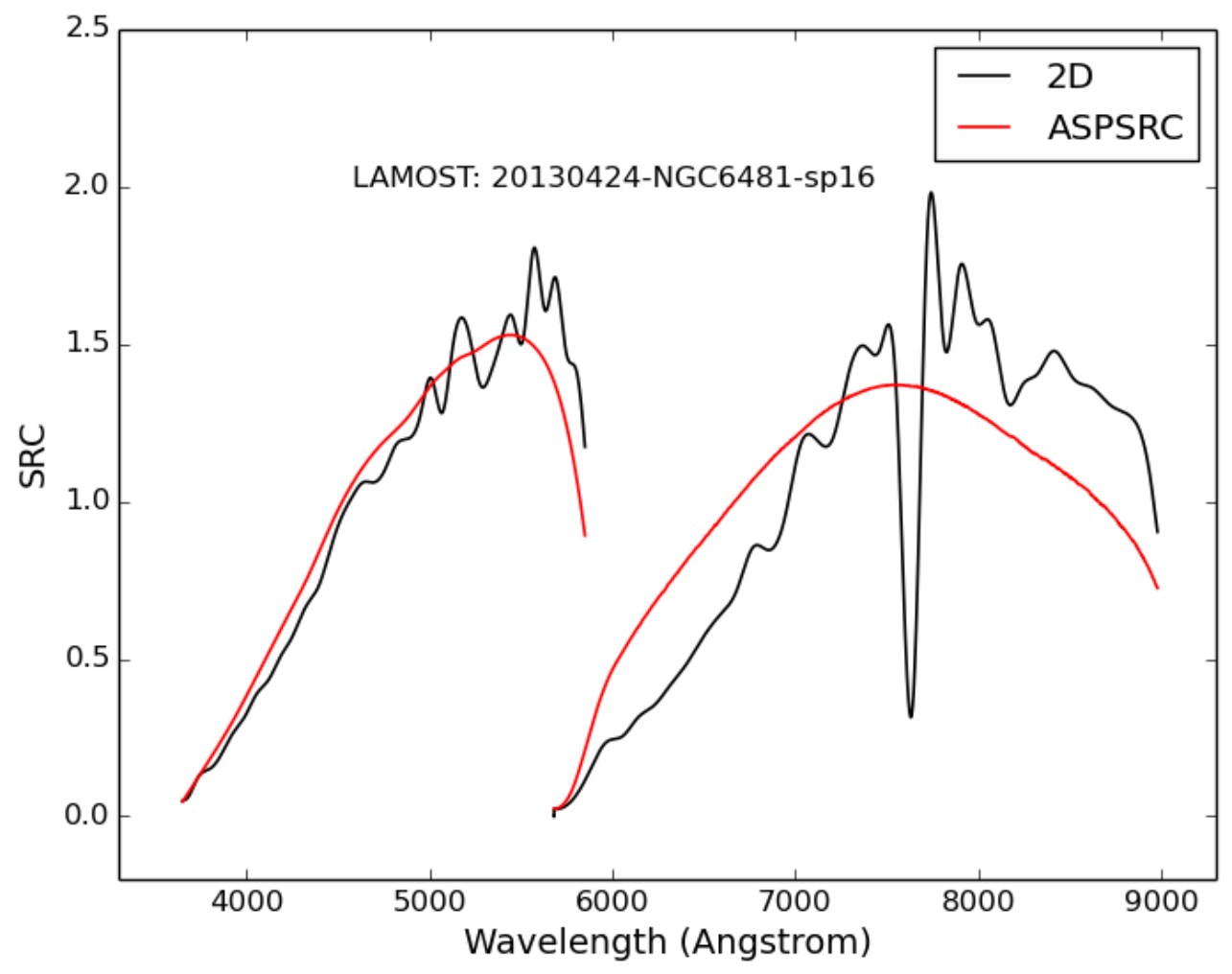

Fig. 21.- Comparison of the over fitted SRCs from the 2D pipeline (black) with the ASPSRCs (red). 


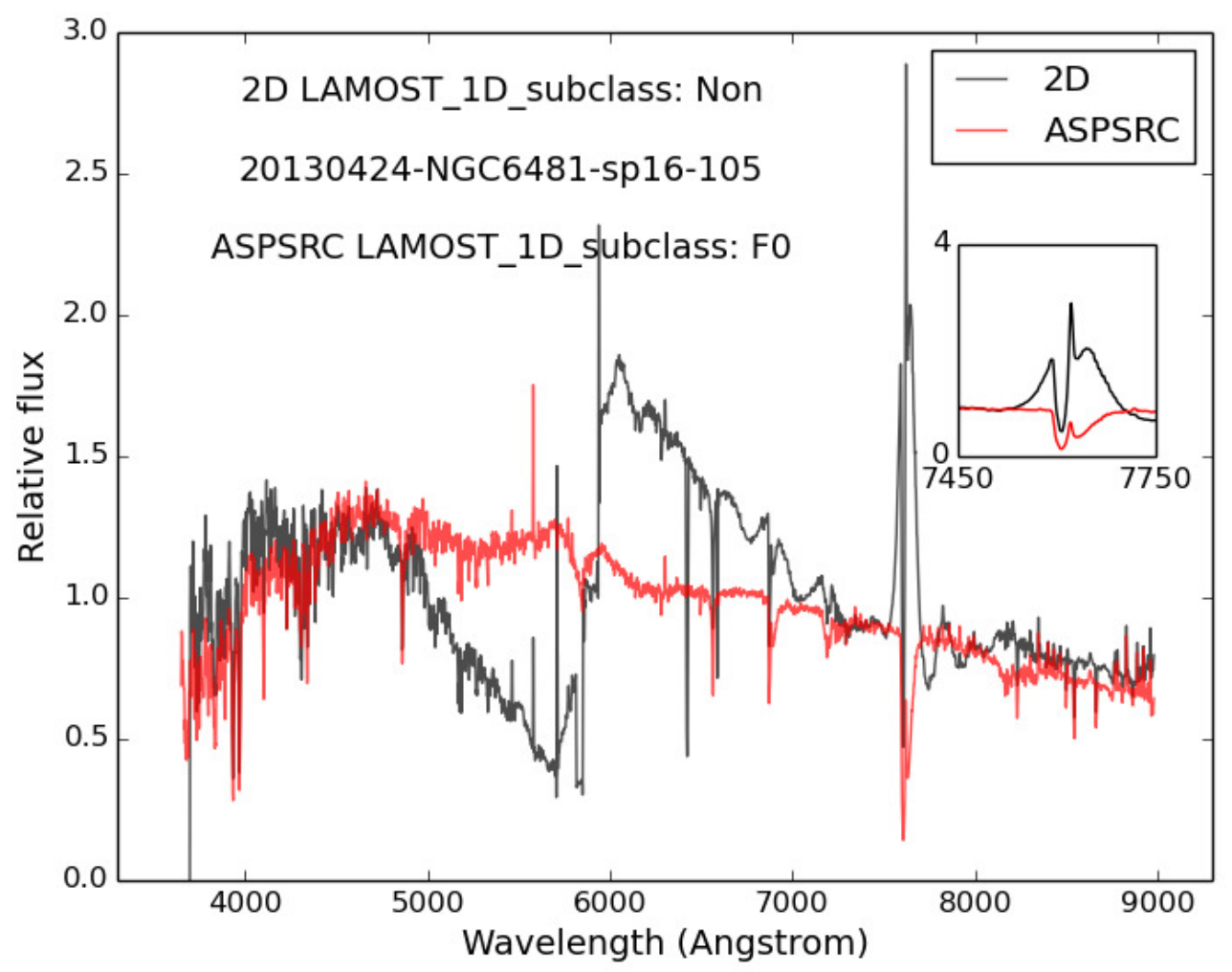

Fig. 22.- Comparison of the artificial spectra calibrated by adopting the over fitted SRCs in Fig 18 (black) with the spectra recalibrated by the ASPSRCs (red). The spectra is classified as an F0 instead of "Non" after recalibration. 
Table 1: The absolute and relative uncertainties of the $\mathrm{g} r$ i regions.

\begin{tabular}{lllllll} 
No-spectrograph & g-absolute & g-relative & r-absolute & r-relative & i-absolute & i-relative \\
\hline \hline sp01 & 0.054 & $6.35 \%$ & 0.054 & $6.75 \%$ & 0.033 & $2.83 \%$ \\
sp02 & 0.046 & $5.12 \%$ & 0.045 & $5.96 \%$ & 0.028 & $2.41 \%$ \\
sp03 & 0.047 & $4.71 \%$ & 0.033 & $6.34 \%$ & 0.031 & $2.74 \%$ \\
sp04 & 0.048 & $5.49 \%$ & 0.039 & $6.74 \%$ & 0.031 & $2.72 \%$ \\
sp05 & 0.053 & $6.95 \%$ & 0.065 & $11.13 \%$ & 0.040 & $3.51 \%$ \\
sp06 & 0.051 & $5.07 \%$ & 0.049 & $7.83 \%$ & 0.035 & $3.20 \%$ \\
sp07 & 0.048 & $5.22 \%$ & 0.046 & $6.21 \%$ & 0.031 & $2.71 \%$ \\
sp08 & 0.052 & $6.30 \%$ & 0.052 & $6.91 \%$ & 0.038 & $3.38 \%$ \\
sp09 & 0.053 & $6.57 \%$ & 0.051 & $6.46 \%$ & 0.031 & $2.74 \%$ \\
sp10 & 0.046 & $5.25 \%$ & 0.046 & $7.32 \%$ & 0.039 & $3.60 \%$ \\
sp11 & 0.045 & $5.57 \%$ & 0.036 & $6.70 \%$ & 0.033 & $2.93 \%$ \\
sp12 & 0.054 & $6.71 \%$ & 0.047 & $7.88 \%$ & 0.047 & $4.11 \%$ \\
sp13 & 0.053 & $6.44 \%$ & 0.041 & $6.94 \%$ & 0.034 & $2.99 \%$ \\
sp14 & 0.048 & $5.97 \%$ & 0.039 & $6.74 \%$ & 0.033 & $2.83 \%$ \\
sp15 & 0.041 & $5.12 \%$ & 0.035 & $5.82 \%$ & 0.029 & $2.60 \%$ \\
sp16 & 0.041 & $5.02 \%$ & 0.034 & $5.66 \%$ & 0.032 & $2.82 \%$ \\
\hline
\end{tabular}


Table 2: The final released spectral classifications of the abandoned spectrographs.

\begin{tabular}{ll} 
Type & Number \\
\hline \hline total 52181 & \\
Galaxy & 1163 \\
QSO & 201 \\
BA & 1477 \\
F & 8454 \\
G & 13875 \\
K & 14440 \\
M & 12571 \\
\hline
\end{tabular}


Table 3:: The blue-arm ASPSRCs of LAMOST spectrographs from No.1 to No.8 at 100

Åsteps. All the ASPSRCs are scaled to a mean value of unity.

\begin{tabular}{|c|c|c|c|c|c|c|c|c|}
\hline & No.1 & No.2 & No. 3 & No.4 & No.5 & No. 6 & No.7 & No. 8 \\
\hline Wavelength & SRC(Err) & $\mathrm{SRC}(\mathrm{Err})$ & SRC(Err) & $\mathrm{SRC}(\mathrm{Err})$ & SRC(Err) & $\mathrm{SRC}(\mathrm{Err})$ & $\mathrm{SRC}(\mathrm{Err})$ & $\mathrm{SRC}(\mathrm{Err})$ \\
\hline 3650 & $0.142(0.036)$ & $0.132(0.027)$ & $0.106(0.016)$ & $0.070(0.026)$ & $0.054(0.054)$ & $0.150(0.027)$ & $0.098(0.016)$ & $0.048(0.053)$ \\
\hline 3750 & $0.245(0.051)$ & $0.244(0.039)$ & $0.261(0.040)$ & $0.187(0.030)$ & $0.122(0.046)$ & $0.268(0.044)$ & $0.202(0.034)$ & $0.156(0.029)$ \\
\hline 3850 & $0.351(0.063)$ & $0.356(0.048)$ & $0.409(0.051)$ & $0.307(0.041)$ & $0.207(0.035)$ & $0.397(0.056)$ & $0.325(0.049)$ & $0.267(0.039)$ \\
\hline 3950 & $0.460(0.070)$ & $0.467(0.054)$ & $0.550(0.058)$ & $0.429(0.050)$ & $0.305(0.043)$ & $0.531(0.065)$ & $0.455(0.058)$ & $0.379(0.048)$ \\
\hline 4050 & $0.570(0.076)$ & $0.578(0.060)$ & $0.682(0.063)$ & $0.551(0.059)$ & $0.415(0.069)$ & $0.670(0.072)$ & $0.586(0.068)$ & $0.491(0.059)$ \\
\hline 4150 & $0.680(0.077)$ & $0.687(0.061)$ & $0.805(0.065)$ & $0.671(0.062)$ & $0.534(0.053)$ & $0.808(0.073)$ & $0.716(0.072)$ & $0.602(0.063)$ \\
\hline 4250 & $0.789(0.075)$ & $0.795(0.059)$ & $0.917(0.066)$ & $0.789(0.063)$ & $0.659(0.071)$ & $0.943(0.072)$ & $0.844(0.072)$ & $0.710(0.064)$ \\
\hline 4350 & $0.896(0.069)$ & $0.900(0.054)$ & $1.022(0.061)$ & $0.903(0.060)$ & $0.788(0.084)$ & $1.073(0.067)$ & $0.973(0.064)$ & $0.820(0.064)$ \\
\hline 4450 & $1.005(0.064)$ & $1.007(0.050)$ & $1.124(0.057)$ & $1.010(0.059)$ & $0.919(0.054)$ & $1.197(0.060)$ & $1.110(0.057)$ & $0.942(0.063)$ \\
\hline 4550 & $1.120(0.056)$ & $1.130(0.043)$ & $1.224(0.049)$ & $1.110(0.054)$ & $1.050(0.051)$ & $1.302(0.048)$ & $1.251(0.048)$ & $1.072(0.058)$ \\
\hline 4650 & $1.190(0.046)$ & $1.207(0.037)$ & $1.294(0.041)$ & $1.201(0.047)$ & $1.174(0.045)$ & $1.374(0.038)$ & $1.359(0.038)$ & $1.157(0.053)$ \\
\hline 4750 & $1.270(0.037)$ & $1.288(0.033)$ & $1.339(0.031)$ & $1.281(0.036)$ & $1.281(0.037)$ & $1.446(0.031)$ & $1.476(0.028)$ & $1.277(0.044)$ \\
\hline 4850 & $1.353(0.026)$ & $1.400(0.033)$ & $1.363(0.022)$ & $1.344(0.022)$ & $1.363(0.032)$ & $1.493(0.028)$ & $1.565(0.016)$ & $1.396(0.032)$ \\
\hline 4950 & $1.423(0.016)$ & $1.511(0.034)$ & $1.394(0.015)$ & $1.372(0.013)$ & $1.434(0.036)$ & $1.514(0.027)$ & $1.634(0.014)$ & $1.492(0.019)$ \\
\hline 5050 & $1.484(0.022)$ & $1.604(0.031)$ & $1.432(0.020)$ & $1.417(0.018)$ & $1.507(0.030)$ & $1.533(0.029)$ & $1.702(0.026)$ & $1.572(0.014)$ \\
\hline 5150 & $1.538(0.046)$ & $1.667(0.032)$ & $1.429(0.030)$ & $1.456(0.028)$ & $1.577(0.038)$ & $1.554(0.039)$ & $1.782(0.040)$ & $1.683(0.031)$ \\
\hline 5250 & $1.584(0.065)$ & $1.705(0.042)$ & $1.383(0.039)$ & $1.477(0.038)$ & $1.608(0.050)$ & $1.543(0.051)$ & $1.788(0.056)$ & $1.775(0.050)$ \\
\hline 5350 & $1.618(0.082)$ & $1.721(0.064)$ & $1.336(0.050)$ & $1.472(0.047)$ & $1.613(0.062)$ & $1.515(0.064)$ & $1.773(0.073)$ & $1.814(0.071)$ \\
\hline 5450 & $1.626(0.091)$ & $1.685(0.085)$ & $1.280(0.059)$ & $1.442(0.057)$ & $1.605(0.077)$ & $1.455(0.073)$ & $1.681(0.087)$ & $1.797(0.088)$ \\
\hline 5550 & $1.564(0.090)$ & $1.536(0.096)$ & $1.185(0.067)$ & $1.394(0.067)$ & $1.571(0.099)$ & $1.303(0.079)$ & $1.331(0.088)$ & $1.683(0.093)$ \\
\hline 5650 & $1.340(0.084)$ & $1.205(0.092)$ & $1.068(0.070)$ & $1.329(0.074)$ & $1.498(0.111)$ & $0.979(0.085)$ & $0.769(0.064)$ & $1.409(0.080)$ \\
\hline 5750 & $0.845(0.082)$ & $0.604(0.076)$ & $0.928(0.068)$ & $1.229(0.077)$ & $1.351(0.097)$ & $0.430(0.074)$ & $0.257(0.028)$ & $0.853(0.054)$ \\
\hline 5850 & $0.114(0.017)$ & $0.095(0.017)$ & $0.749(0.063)$ & $1.060(0.075)$ & $0.977(0.272)$ & $0.134(0.034)$ & $0.007(0.000)$ & $0.127(0.009)$ \\
\hline
\end{tabular}


Table 4:: The red-arm ASPSRCs of LAMOST spectrographs from No.1 to No.8 at 100 Åsteps.

\begin{tabular}{|c|c|c|c|c|c|c|c|c|}
\hline & No.1 & No. 2 & No.3 & No.4 & No.5 & No.6 & No.7 & No. 8 \\
\hline Wavelength & $\mathrm{SRC}(\mathrm{Err})$ & $\mathrm{SRC}$ (Err) & $\mathrm{SRC}(\mathrm{Err})$ & $\mathrm{SRC}(\operatorname{Err})$ & SRC(Err) & $\mathrm{SRC}(\mathrm{Err})$ & $\mathrm{SRC}(\mathrm{Err})$ & $\mathrm{SRC}(\operatorname{Err})$ \\
\hline 5680 & $0.070(0.047)$ & $0.116(0.030)$ & $-0.005(0.003)$ & $0.006(0.009)$ & $-0.018(0.008)$ & $0.185(0.057)$ & $0.329(0.065)$ & $0.081(0.032)$ \\
\hline 5780 & $0.333(0.047)$ & $0.330(0.045)$ & $0.031(0.007)$ & $0.044(0.007)$ & $0.074(0.070)$ & $0.269(0.049)$ & $0.433(0.049)$ & $0.273(0.035)$ \\
\hline 5880 & $0.509(0.062)$ & $0.478(0.049)$ & $0.140(0.014)$ & $0.187(0.020)$ & $0.273(0.088)$ & $0.356(0.053)$ & $0.520(0.053)$ & $0.423(0.056)$ \\
\hline 5980 & $0.620(0.065)$ & $0.569(0.046)$ & $0.283(0.028)$ & $0.371(0.044)$ & $0.458(0.091)$ & $0.436(0.054)$ & $0.601(0.053)$ & $0.537(0.065)$ \\
\hline 6080 & $0.696(0.064)$ & $0.639(0.046)$ & $0.427(0.039)$ & $0.545(0.061)$ & $0.585(0.088)$ & $0.513(0.057)$ & $0.674(0.054)$ & $0.626(0.069)$ \\
\hline 6180 & $0.760(0.062)$ & $0.711(0.047)$ & $0.543(0.044)$ & $0.675(0.066)$ & $0.672(0.079)$ & $0.587(0.059)$ & $0.748(0.054)$ & $0.703(0.069)$ \\
\hline 6280 & $0.820(0.056)$ & $0.779(0.046)$ & $0.634(0.046)$ & $0.763(0.065)$ & $0.745(0.077)$ & $0.659(0.059)$ & $0.817(0.053)$ & $0.770(0.068)$ \\
\hline 6380 & $0.882(0.053)$ & $0.855(0.046)$ & $0.715(0.049)$ & $0.834(0.062)$ & $0.815(0.078)$ & $0.735(0.061)$ & $0.892(0.054)$ & $0.842(0.067)$ \\
\hline 6480 & $0.938(0.048)$ & $0.929(0.044)$ & $0.791(0.050)$ & $0.895(0.058)$ & $0.881(0.070)$ & $0.810(0.059)$ & $0.960(0.052)$ & $0.905(0.063)$ \\
\hline 6580 & $0.995(0.044)$ & $1.001(0.042)$ & $0.867(0.048)$ & $0.956(0.053)$ & $0.949(0.064)$ & $0.884(0.057)$ & $1.024(0.049)$ & $0.970(0.059)$ \\
\hline 6680 & $1.052(0.041)$ & $1.071(0.039)$ & $0.943(0.046)$ & $1.019(0.047)$ & $1.020(0.062)$ & $0.956(0.054)$ & $1.086(0.047)$ & $1.035(0.055)$ \\
\hline 6780 & $1.104(0.038)$ & $1.137(0.035)$ & $1.019(0.044)$ & $1.080(0.041)$ & $1.086(0.051)$ & $1.026(0.049)$ & $1.141(0.043)$ & $1.100(0.050)$ \\
\hline 6880 & $1.154(0.034)$ & $1.198(0.033)$ & $1.091(0.041)$ & $1.138(0.034)$ & $1.151(0.042)$ & $1.091(0.044)$ & $1.192(0.037)$ & $1.161(0.046)$ \\
\hline 6980 & $1.196(0.033)$ & $1.250(0.028)$ & $1.157(0.038)$ & $1.190(0.032)$ & $1.207(0.034)$ & $1.152(0.036)$ & $1.235(0.032)$ & $1.212(0.038)$ \\
\hline 7080 & $1.234(0.032)$ & $1.297(0.024)$ & $1.219(0.034)$ & $1.239(0.026)$ & $1.258(0.031)$ & $1.207(0.029)$ & $1.273(0.026)$ & $1.261(0.033)$ \\
\hline 7180 & $1.265(0.029)$ & $1.334(0.021)$ & $1.273(0.030)$ & $1.279(0.022)$ & $1.300(0.031)$ & $1.254(0.022)$ & $1.302(0.021)$ & $1.298(0.028)$ \\
\hline 7280 & $1.288(0.029)$ & $1.362(0.019)$ & $1.320(0.027)$ & $1.312(0.021)$ & $1.332(0.030)$ & $1.291(0.019)$ & $1.323(0.018)$ & $1.328(0.025)$ \\
\hline 7380 & $1.304(0.025)$ & $1.379(0.014)$ & $1.357(0.018)$ & $1.336(0.013)$ & $1.353(0.024)$ & $1.319(0.012)$ & $1.333(0.010)$ & $1.349(0.021)$ \\
\hline 7480 & $1.312(0.025)$ & $1.384(0.013)$ & $1.384(0.014)$ & $1.350(0.015)$ & $1.366(0.022)$ & $1.339(0.012)$ & $1.336(0.010)$ & $1.361(0.019)$ \\
\hline 7580 & $1.313(0.023)$ & $1.378(0.015)$ & $1.401(0.011)$ & $1.355(0.020)$ & $1.366(0.024)$ & $1.349(0.015)$ & $1.329(0.012)$ & $1.361(0.019)$ \\
\hline 7680 & $1.305(0.024)$ & $1.363(0.017)$ & $1.407(0.014)$ & $1.350(0.022)$ & $1.356(0.028)$ & $1.351(0.020)$ & $1.313(0.018)$ & $1.348(0.022)$ \\
\hline 7780 & $1.291(0.023)$ & $1.339(0.021)$ & $1.407(0.018)$ & $1.338(0.026)$ & $1.337(0.028)$ & $1.345(0.024)$ & $1.287(0.021)$ & $1.327(0.023)$ \\
\hline 7880 & $1.271(0.024)$ & $1.308(0.021)$ & $1.399(0.018)$ & $1.318(0.026)$ & $1.313(0.030)$ & $1.331(0.029)$ & $1.255(0.025)$ & $1.298(0.026)$ \\
\hline 7980 & $1.246(0.028)$ & $1.269(0.025)$ & $1.385(0.022)$ & $1.291(0.027)$ & $1.286(0.032)$ & $1.313(0.034)$ & $1.218(0.029)$ & $1.261(0.031)$ \\
\hline 8080 & $1.218(0.033)$ & $1.230(0.030)$ & $1.366(0.025)$ & $1.264(0.029)$ & $1.252(0.041)$ & $1.292(0.041)$ & $1.178(0.034)$ & $1.216(0.038)$ \\
\hline 8180 & $1.180(0.035)$ & $1.186(0.036)$ & $1.342(0.031)$ & $1.232(0.030)$ & $1.220(0.046)$ & $1.267(0.045)$ & $1.130(0.040)$ & $1.171(0.050)$ \\
\hline 8280 & $1.139(0.041)$ & $1.139(0.043)$ & $1.313(0.037)$ & $1.198(0.031)$ & $1.187(0.052)$ & $1.235(0.053)$ & $1.082(0.044)$ & $1.120(0.061)$ \\
\hline 8380 & $1.101(0.041)$ & $1.092(0.042)$ & $1.286(0.043)$ & $1.170(0.038)$ & $1.158(0.047)$ & $1.203(0.052)$ & $1.032(0.046)$ & $1.081(0.056)$ \\
\hline 8480 & $1.062(0.045)$ & $1.042(0.048)$ & $1.256(0.047)$ & $1.142(0.044)$ & $1.128(0.046)$ & $1.163(0.053)$ & $0.980(0.049)$ & $1.034(0.061)$ \\
\hline 8580 & $1.014(0.045)$ & $0.986(0.050)$ & $1.222(0.051)$ & $1.117(0.045)$ & $1.091(0.048)$ & $1.116(0.055)$ & $0.925(0.051)$ & $0.977(0.065)$ \\
\hline 8680 & $0.962(0.046)$ & $0.925(0.051)$ & $1.182(0.054)$ & $1.091(0.047)$ & $1.048(0.053)$ & $1.060(0.053)$ & $0.868(0.052)$ & $0.921(0.068)$ \\
\hline 8780 & $0.907(0.046)$ & $0.853(0.049)$ & $1.125(0.055)$ & $1.047(0.050)$ & $0.990(0.053)$ & $0.991(0.051)$ & $0.806(0.051)$ & $0.855(0.067)$ \\
\hline 8880 & $0.841(0.050)$ & $0.773(0.049)$ & $1.037(0.059)$ & $0.972(0.054)$ & $0.916(0.056)$ & $0.911(0.052)$ & $0.740(0.054)$ & $0.778(0.064)$ \\
\hline 8980 & $0.757(0.044)$ & $0.677(0.044)$ & $0.898(0.053)$ & $0.840(0.043)$ & $0.794(0.060)$ & $0.820(0.052)$ & $0.670(0.051)$ & $0.673(0.069)$ \\
\hline
\end{tabular}


Table 5:: The blue-arm ASPSRCs of LAMOST spectrographs from No.9 to No.16 at 100 Åsteps.

\begin{tabular}{|c|c|c|c|c|c|c|c|c|}
\hline & No.9 & No.10 & No.11 & No.12 & No.13 & No.14 & No.15 & No.16 \\
\hline Wavelength & $\mathrm{SRC}(\mathrm{Err})$ & $\mathrm{SRC}(\operatorname{Err})$ & $\mathrm{SRC}(\mathrm{Err})$ & SRC(Err) & $\mathrm{SRC}($ Err) & $\mathrm{SRC}($ Err) & SRC(Err) & SRC(Err) \\
\hline 3650 & $0.080(0.012)$ & $0.096(0.019)$ & $0.082(0.014)$ & $0.027(0.006)$ & $0.058(0.027)$ & $0.069(0.023)$ & $0.013(0.037)$ & $0.049(0.018)$ \\
\hline 3750 & $0.160(0.027)$ & $0.201(0.036)$ & $0.179(0.025)$ & $0.122(0.019)$ & $0.106(0.017)$ & $0.132(0.028)$ & $0.154(0.031)$ & $0.144(0.020)$ \\
\hline 3850 & $0.254(0.038)$ & $0.309(0.046)$ & $0.275(0.033)$ & $0.228(0.032)$ & $0.171(0.023)$ & $0.199(0.033)$ & $0.272(0.040)$ & $0.241(0.029)$ \\
\hline 3950 & $0.359(0.046)$ & $0.428(0.054)$ & $0.375(0.039)$ & $0.338(0.042)$ & $0.249(0.031)$ & $0.277(0.041)$ & $0.390(0.045)$ & $0.347(0.036)$ \\
\hline 4050 & $0.470(0.056)$ & $0.557(0.061)$ & $0.480(0.046)$ & $0.449(0.051)$ & $0.338(0.039)$ & $0.372(0.048)$ & $0.509(0.051)$ & $0.463(0.044)$ \\
\hline 4150 & $0.581(0.063)$ & $0.686(0.064)$ & $0.587(0.051)$ & $0.559(0.056)$ & $0.435(0.042)$ & $0.479(0.052)$ & $0.622(0.053)$ & $0.583(0.049)$ \\
\hline 4250 & $0.690(0.068)$ & $0.802(0.066)$ & $0.694(0.054)$ & $0.666(0.063)$ & $0.536(0.045)$ & $0.589(0.055)$ & $0.716(0.053)$ & $0.699(0.051)$ \\
\hline 4350 & $0.805(0.067)$ & $0.923(0.062)$ & $0.806(0.056)$ & $0.779(0.064)$ & $0.649(0.048)$ & $0.709(0.055)$ & $0.801(0.049)$ & $0.822(0.051)$ \\
\hline 4450 & $0.936(0.067)$ & $1.066(0.058)$ & $0.930(0.057)$ & $0.908(0.065)$ & $0.782(0.054)$ & $0.844(0.055)$ & $0.908(0.046)$ & $0.961(0.051)$ \\
\hline 4550 & $1.061(0.062)$ & $1.185(0.050)$ & $1.045(0.054)$ & $1.029(0.065)$ & $0.912(0.059)$ & $0.973(0.053)$ & $1.013(0.041)$ & $1.084(0.046)$ \\
\hline 4650 & $1.161(0.051)$ & $1.272(0.040)$ & $1.136(0.048)$ & $1.138(0.060)$ & $1.033(0.061)$ & $1.085(0.049)$ & $1.104(0.036)$ & $1.181(0.040)$ \\
\hline 4750 & $1.247(0.040)$ & $1.344(0.029)$ & $1.209(0.039)$ & $1.235(0.052)$ & $1.148(0.061)$ & $1.186(0.046)$ & $1.184(0.031)$ & $1.255(0.032)$ \\
\hline 4850 & $1.346(0.029)$ & $1.417(0.018)$ & $1.284(0.030)$ & $1.319(0.040)$ & $1.265(0.056)$ & $1.293(0.042)$ & $1.265(0.027)$ & $1.314(0.026)$ \\
\hline 4950 & $1.444(0.026)$ & $1.497(0.015)$ & $1.369(0.022)$ & $1.383(0.027)$ & $1.400(0.049)$ & $1.399(0.040)$ & $1.354(0.023)$ & $1.395(0.020)$ \\
\hline 5050 & $1.522(0.033)$ & $1.558(0.016)$ & $1.439(0.013)$ & $1.443(0.012)$ & $1.517(0.038)$ & $1.492(0.036)$ & $1.443(0.022)$ & $1.473(0.017)$ \\
\hline 5150 & $1.574(0.048)$ & $1.597(0.027)$ & $1.472(0.017)$ & $1.508(0.016)$ & $1.586(0.025)$ & $1.561(0.033)$ & $1.512(0.024)$ & $1.528(0.018)$ \\
\hline 5250 & $1.615(0.054)$ & $1.592(0.039)$ & $1.475(0.026)$ & $1.536(0.034)$ & $1.636(0.025)$ & $1.590(0.034)$ & $1.539(0.032)$ & $1.558(0.025)$ \\
\hline 5350 & $1.660(0.062)$ & $1.606(0.055)$ & $1.478(0.040)$ & $1.536(0.054)$ & $1.697(0.037)$ & $1.605(0.039)$ & $1.570(0.040)$ & $1.597(0.037)$ \\
\hline 5450 & $1.682(0.073)$ & $1.595(0.069)$ & $1.474(0.054)$ & $1.528(0.070)$ & $1.736(0.056)$ & $1.614(0.049)$ & $1.576(0.049)$ & $1.612(0.050)$ \\
\hline 5550 & $1.619(0.088)$ & $1.497(0.081)$ & $1.441(0.067)$ & $1.508(0.081)$ & $1.705(0.076)$ & $1.591(0.061)$ & $1.526(0.057)$ & $1.586(0.064)$ \\
\hline 5650 & $1.431(0.094)$ & $1.289(0.088)$ & $1.385(0.079)$ & $1.472(0.088)$ & $1.634(0.092)$ & $1.545(0.073)$ & $1.442(0.062)$ & $1.501(0.075)$ \\
\hline 5750 & $1.069(0.087)$ & $0.873(0.075)$ & $1.295(0.088)$ & $1.403(0.095)$ & $1.514(0.106)$ & $1.456(0.081)$ & $1.305(0.066)$ & $1.308(0.078)$ \\
\hline 5850 & $0.524(0.060)$ & $0.168(0.019)$ & $1.147(0.089)$ & $1.283(0.097)$ & $1.302(0.111)$ & $1.290(0.086)$ & $1.078(0.067)$ & $0.941(0.068)$ \\
\hline
\end{tabular}


Table 6:: The red-arm ASPSRCs of LAMOST spectrographs from No.9 to No.16 at 100 Åsteps.

\begin{tabular}{|c|c|c|c|c|c|c|c|c|}
\hline & No.9 & No.10 & No.11 & No.12 & No.13 & No.14 & No.15 & No.16 \\
\hline Wavelength & SRC(Err) & SRC(Err) & SRC(Err) & SRC(Err) & SRC(Err) & SRC(Err) & SRC(Err) & SRC(Err) \\
\hline 5680 & $-0.004(0.088)$ & $0.027(0.015)$ & $0.007(0.033)$ & $0.013(0.005)$ & $0.017(0.049)$ & $0.004(0.000)$ & $-0.013(0.041)$ & $0.024(0.008)$ \\
\hline 5780 & $0.251(0.031)$ & $0.198(0.028)$ & $0.041(0.007)$ & $0.027(0.005)$ & $0.065(0.012)$ & $0.045(0.007)$ & $0.086(0.010)$ & $0.085(0.012)$ \\
\hline 5880 & $0.508(0.063)$ & $0.340(0.048)$ & $0.159(0.021)$ & $0.136(0.020)$ & $0.251(0.034)$ & $0.183(0.022)$ & $0.254(0.031)$ & $0.275(0.031)$ \\
\hline 5980 & $0.665(0.067)$ & $0.437(0.055)$ & $0.330(0.039)$ & $0.329(0.045)$ & $0.482(0.055)$ & $0.370(0.041)$ & $0.416(0.046)$ & $0.447(0.045)$ \\
\hline 6080 & $0.749(0.068)$ & $0.517(0.060)$ & $0.501(0.054)$ & $0.524(0.067)$ & $0.630(0.062)$ & $0.551(0.053)$ & $0.556(0.054)$ & $0.552(0.048)$ \\
\hline 6180 & $0.814(0.063)$ & $0.590(0.063)$ & $0.633(0.060)$ & $0.656(0.078)$ & $0.731(0.064)$ & $0.691(0.058)$ & $0.666(0.055)$ & $0.643(0.051)$ \\
\hline 6280 & $0.873(0.059)$ & $0.659(0.065)$ & $0.726(0.059)$ & $0.732(0.080)$ & $0.808(0.061)$ & $0.790(0.059)$ & $0.751(0.054)$ & $0.722(0.051)$ \\
\hline 6380 & $0.942(0.055)$ & $0.726(0.066)$ & $0.807(0.059)$ & $0.811(0.083)$ & $0.873(0.060)$ & $0.872(0.060)$ & $0.826(0.052)$ & $0.801(0.053)$ \\
\hline 6480 & $1.010(0.050)$ & $0.794(0.067)$ & $0.876(0.057)$ & $0.877(0.080)$ & $0.935(0.058)$ & $0.945(0.058)$ & $0.895(0.050)$ & $0.876(0.052)$ \\
\hline 6580 & $1.074(0.047)$ & $0.865(0.064)$ & $0.945(0.054)$ & $0.939(0.076)$ & $0.995(0.055)$ & $1.017(0.054)$ & $0.965(0.047)$ & $0.946(0.050)$ \\
\hline 6680 & $1.138(0.044)$ & $0.938(0.061)$ & $1.016(0.051)$ & $1.013(0.073)$ & $1.056(0.051)$ & $1.087(0.051)$ & $1.037(0.044)$ & $1.019(0.048)$ \\
\hline 6780 & $1.193(0.039)$ & $1.012(0.056)$ & $1.087(0.047)$ & $1.084(0.068)$ & $1.112(0.047)$ & $1.153(0.046)$ & $1.110(0.041)$ & $1.088(0.045)$ \\
\hline 6880 & $1.244(0.035)$ & $1.085(0.050)$ & $1.155(0.043)$ & $1.156(0.065)$ & $1.164(0.042)$ & $1.217(0.042)$ & $1.177(0.037)$ & $1.153(0.042)$ \\
\hline 6980 & $1.281(0.029)$ & $1.154(0.045)$ & $1.213(0.037)$ & $1.214(0.056)$ & $1.207(0.039)$ & $1.272(0.038)$ & $1.239(0.034)$ & $1.210(0.037)$ \\
\hline 7080 & $1.315(0.025)$ & $1.217(0.038)$ & $1.267(0.033)$ & $1.267(0.049)$ & $1.242(0.034)$ & $1.319(0.031)$ & $1.294(0.028)$ & $1.261(0.032)$ \\
\hline 7180 & $1.337(0.022)$ & $1.271(0.032)$ & $1.312(0.028)$ & $1.317(0.041)$ & $1.270(0.029)$ & $1.357(0.026)$ & $1.338(0.024)$ & $1.307(0.027)$ \\
\hline 7280 & $1.347(0.020)$ & $1.314(0.026)$ & $1.344(0.025)$ & $1.354(0.035)$ & $1.293(0.027)$ & $1.385(0.023)$ & $1.371(0.022)$ & $1.342(0.023)$ \\
\hline 7380 & $1.350(0.017)$ & $1.345(0.017)$ & $1.369(0.019)$ & $1.383(0.025)$ & $1.315(0.018)$ & $1.405(0.015)$ & $1.392(0.014)$ & $1.368(0.016)$ \\
\hline 7480 & $1.345(0.021)$ & $1.363(0.014)$ & $1.382(0.016)$ & $1.402(0.017)$ & $1.333(0.014)$ & $1.416(0.011)$ & $1.399(0.010)$ & $1.383(0.013)$ \\
\hline 7580 & $1.331(0.023)$ & $1.369(0.012)$ & $1.386(0.013)$ & $1.409(0.013)$ & $1.337(0.014)$ & $1.412(0.014)$ & $1.394(0.011)$ & $1.386(0.012)$ \\
\hline 7680 & $1.307(0.023)$ & $1.365(0.016)$ & $1.381(0.014)$ & $1.407(0.016)$ & $1.333(0.019)$ & $1.397(0.018)$ & $1.379(0.015)$ & $1.377(0.016)$ \\
\hline 7780 & $1.271(0.028)$ & $1.353(0.022)$ & $1.366(0.020)$ & $1.393(0.021)$ & $1.320(0.023)$ & $1.368(0.022)$ & $1.353(0.017)$ & $1.360(0.020)$ \\
\hline 7880 & $1.227(0.024)$ & $1.333(0.026)$ & $1.344(0.019)$ & $1.367(0.030)$ & $1.298(0.025)$ & $1.330(0.025)$ & $1.322(0.021)$ & $1.333(0.022)$ \\
\hline 7980 & $1.180(0.026)$ & $1.309(0.034)$ & $1.319(0.025)$ & $1.338(0.038)$ & $1.267(0.029)$ & $1.285(0.030)$ & $1.283(0.025)$ & $1.300(0.027)$ \\
\hline 8080 & $1.131(0.027)$ & $1.282(0.041)$ & $1.290(0.032)$ & $1.296(0.049)$ & $1.228(0.033)$ & $1.236(0.034)$ & $1.243(0.032)$ & $1.262(0.033)$ \\
\hline 8180 & $1.078(0.035)$ & $1.254(0.051)$ & $1.255(0.040)$ & $1.244(0.057)$ & $1.185(0.036)$ & $1.183(0.039)$ & $1.202(0.036)$ & $1.220(0.038)$ \\
\hline 8280 & $1.024(0.044)$ & $1.227(0.055)$ & $1.215(0.048)$ & $1.183(0.062)$ & $1.140(0.037)$ & $1.130(0.041)$ & $1.160(0.042)$ & $1.176(0.045)$ \\
\hline 8380 & $0.982(0.048)$ & $1.200(0.064)$ & $1.179(0.051)$ & $1.137(0.062)$ & $1.096(0.046)$ & $1.080(0.045)$ & $1.120(0.043)$ & $1.141(0.046)$ \\
\hline 8480 & $0.937(0.052)$ & $1.164(0.068)$ & $1.140(0.055)$ & $1.090(0.064)$ & $1.054(0.050)$ & $1.033(0.048)$ & $1.079(0.047)$ & $1.102(0.051)$ \\
\hline 8580 & $0.888(0.062)$ & $1.121(0.071)$ & $1.093(0.057)$ & $1.046(0.069)$ & $1.013(0.052)$ & $0.989(0.048)$ & $1.039(0.048)$ & $1.055(0.051)$ \\
\hline 8680 & $0.828(0.070)$ & $1.070(0.074)$ & $1.040(0.062)$ & $0.992(0.071)$ & $0.969(0.054)$ & $0.945(0.051)$ & $0.994(0.050)$ & $0.998(0.052)$ \\
\hline 8780 & $0.756(0.072)$ & $1.005(0.071)$ & $0.971(0.060)$ & $0.936(0.072)$ & $0.911(0.058)$ & $0.892(0.049)$ & $0.931(0.049)$ & $0.933(0.052)$ \\
\hline 8880 & $0.672(0.078)$ & $0.920(0.078)$ & $0.883(0.063)$ & $0.854(0.074)$ & $0.828(0.063)$ & $0.815(0.053)$ & $0.851(0.055)$ & $0.853(0.051)$ \\
\hline 8980 & $0.562(0.079)$ & $0.787(0.062)$ & $0.754(0.056)$ & $0.716(0.070)$ & $0.700(0.047)$ & $0.693(0.047)$ & $0.720(0.046)$ & $0.734(0.048)$ \\
\hline
\end{tabular}

